\title{
Divergent DNA methylation signatures of $X$ chromosome regulation in marsupials and eutherians
}

Devika Singh ${ }^{1}$, Dan Sun $^{1}$, Andrew G. King ${ }^{2}$, David E. Alquezar-Planas², Rebecca N. Johnson ${ }^{2,3}$, David Alvarez-Ponce ${ }^{4}, *$, and Soojin V. $\mathrm{Yi}^{1}{ }^{*}{ }^{*}$

${ }^{1}$ School of Biological Sciences, Georgia Institute of Technology, Atlanta, Georgia, USA;

${ }^{2}$ Australian Museum Research Institute, Australian Museum, Sydney, New South Wales, Australia;

${ }^{3}$ National Museum of Natural History, Smithsonian Institution, Washington, DC, USA;

${ }^{4}$ Department of Biology, University of Nevada Reno, Reno, Nevada, USA.

*Corresponding Authors:

David Alvarez-Ponce

dap@unr.edu

$775-682-5735$

Soojin V. Yi

soojin.yi@biology.gatech.edu

404-385-6084 


\section{Abstract}

$3 \mathrm{X}$ chromosome inactivation (XCI) mediated by differential DNA methylation between sexes is 4 well characterized in eutherian mammals. Although XCI is shared between eutherians and 5 marsupials, the role of DNA methylation in marsupial XCI remains contested. Here we examine genome-wide signatures of DNA methylation from methylation maps across fives tissues from a

7 male and female koala (Phascolarctos cinereus) and present the first whole genome, multi-tissue 8 marsupial "methylome atlas." Using these novel data, we elucidate divergent versus common 9 features of marsupial and eutherian DNA methylation. First, tissue-specific differential DNA 10 methylation in marsupials primarily occurs in gene bodies. Second, females show significant 11 global reduction (hypomethylation) of X chromosome DNA methylation compared to males. We 12 show that this pattern is also observed in eutherians. Third, on average, promoter DNA methylation 13 shows little difference between male and female koala $\mathrm{X}$ chromosomes, a pattern distinct from 14 that of eutherians. Fourth, the sex-specific DNA methylation landscape upstream of $R s x$, the 15 primary $\ln c \mathrm{RNA}$ associated with marsupial XCI, is consistent with the epigenetic regulation of 16 female- (and presumably inactive X chromosome-) specific expression. Finally, we utilize the 17 prominent female X chromosome hypomethylation and classify 98 previously unplaced scaffolds 18 as X-linked, contributing an additional 14.6 Mb (21.5\%) to genomic data annotated as the koala 19 X chromosome. Our work demonstrates evolutionarily divergent pathways leading to functionally 20 conserved patterns of XCI in two deep branches of mammals.

23 Keywords: X chromosome inactivation, marsupial, DNA methylation, RSX, koala, whole genome 24 bisulfite sequencing (WGBS) 


\section{Introduction}

$\mathrm{X}$ chromosome inactivation $(\mathrm{XCI})$ is an iconic example of sex chromosome regulation in which one of the two $\mathrm{X}$ chromosomes in females is silenced, a mechanism thought to adjust the expression levels of X-linked genes (1). Although XCI occurs in both eutherian and marsupial mammals (2), there are several notable differences in XCI between the two lineages. First, in eutherians, the transcription of Xist RNA from the inactive X chromosome is essential for XCI (35). However, the Xist locus is not present in marsupials $(6,7)$. Instead, another $\operatorname{lnc}$ RNA gene, $R s x$, drives marsupial XCI (8). Second, marsupials exhibit 'imprinted' XCI by selectively silencing the paternal X chromosome $(9,10)$. In contrast, XCI in eutherians occurs randomly between the maternally and paternally derived X chromosomes, although paternal XCI has been observed during early rodent development $(11,12)$. Third, while eutherian XCI involves the exclusion of active histone marks and the recruitment of repressive histone marks on the inactive $\mathrm{X}$ chromosome (13), marsupial X chromosomes do not show a consistent pattern $(10,14)$. These differences suggest that evolutionary pathways leading to XCI likely differ between eutherians and marsupials, and that novel insights into the mechanism of XCI can be gained from comparative studies.

The role of DNA methylation on marsupial XCI has been particularly controversial. Immunofluorescent labeling studies observed relative hypomethylation of the inactivate $\mathrm{X}$ chromosome in marsupials $(15,16)$. Other studies found little difference in DNA methylation between active and inactive marsupial X chromosomes $(10,17,18)$. Recently, Waters et al. (19) analyzed reduced representation bisulfite sequencing (RRBS) data of a male and female opossum (Monodelphis domestica) and proposed that female $\mathrm{X}$ chromosomes in marsupials, but not in eutherians, exhibit hypomethylation near the transcription start sites (TSSs). Notably, these previous DNA methylation studies either examined a small number of CpGs or employed methodologies that over-represent promoters and $\mathrm{CpG}$ islands (in case of RRBS, (20)). Since patterns of DNA methylation vary greatly among distinctive genomic regions with different functional consequences, it is necessary to extend our knowledge to unbiased, whole-genome assays of DNA methylation.

We used the whole-genome bisulfite sequencing, which captures the DNA methylation state of every nucleotide in the genome, of the modern koala (Phascolarctos cinereus) to understand the role of DNA methylation on XCI in marsupials and to resolve previous 
controversies. We compared DNA methylation maps of five diverse tissues from a male and a

67 female koala, providing the first multi-tissue, whole genome methylome resource of any marsupial

68 with information on tissue-specific variation of DNA methylation. Utilizing these novel resources,

69 we show distinctive impacts of DNA methylation on tissue-specific gene expression in marsupials,

70 as well as on XCI in eutherians and marsupials. We further classify previously undetected X-linked

71 regions from this key marsupial species using characteristic features of $\mathrm{X}$ chromosome DNA

72 methylation. Our findings provide new insights into the evolutionary pathways leading to

73 functionally convergent yet mechanistically divergent pathways of XCI and regulation of gene

74 expression in two deep branches of mammals.

\section{Results}

\section{Genome-wide patterns of DNA methylation in the modern koala}

78 We generated whole genome bisulfite sequencing (WGBS) data from five tissues (brain, lung, 79 kidney, skeletal muscle, and pancreas) from a male ("Ben," Australian Museum registration 80 M.45022) and female koala ("Pacific Chocolate," Australian Museum registration M.47723). The 81 mean depth of coverage fell between $9.9 \times$ and 14.6× (Supplementary Table 1). Autosome-linked 82 scaffolds displayed GC content ranging between 38.58 and $39.59 \%$. Scaffolds linked to

83 chromosome $\mathrm{X}$ had higher GC contents compared to autosomal scaffolds (Supplementary Table 84 2), similar to (21). We performed a hierarchical clustering analysis and observed a clear clustering 85 of samples by tissue (Fig. 1A) with the pancreas samples exhibiting the most unique methylation 86 signature while the kidney and lung samples share the most similar methylation profiles.

\section{Differential DNA methylation between tissues}

89 We identified shared and tissue-specific differentially methylated regions (DMRs) using BSmooth 90 (22). Tissue-specific DMRs were defined as regions that were differentially methylated in a 91 particular tissue compared to all other tissues in a pairwise analysis, while shared DMRs were 92 those found in multiple tissues (Fig. 1B). Consistent with the results of the clustering analysis, the 93 pancreas samples contained the most tissue-specific DMRs followed by the skeletal muscle, brain, 94 kidney and lung (Fig. 1B). The majority (50-53\%) of tissue-specific DMRs fell in gene bodies

95 (Fig. 1C, Supplementary Fig. 1, and Supplementary Table 3), a significant excess compared to 96 length and GC matched control regions (fold enrichment $(\mathrm{FE})=1.25 \sim 1.44, \mathrm{p}<0.0001$ based on 
97 10,000 bootstraps; Fig. 1C, Supplementary Fig. 1 and Supplementary Table 3). On the other hand,

98 DMRs were significantly depleted in intergenic regions compared to the control regions $(\mathrm{p}<0.05$

99 based on 10,000 bootstraps; Fig. 1C, Supplementary Fig. 1, and Supplementary Table 3). Genes

100 containing tissue-specific DMRs were enriched in specific biological functions (Table 1). For

101 example, brain specific DMRs were linked to genes associated with neural developmental

102 processes such as neurogenesis and central nervous system development.

103 We integrated our methylome data with a previously generated RNA-seq koala

104 transcriptomes (23). Of the 12 tissues surveyed in that study, three tissues (kidney, brain, and lung)

105 overlapped with the tissues examined here. Promoter DNA methylation and gene expression were

106 significantly negatively correlated across the entire genome (Table 2, Supplementary Fig. 2). In

107 contrast, the relationship between gene body DNA methylation and expression was complex; both

108 extremely hypomethylated and hypermethylated gene bodies showed high gene expression (Table

109 2, Supplementary Fig. 2). We considered differentially methylated genes (DMGs) containing

110 DMRs between the brain and kidney samples $(n=1944$ genes from $n=4,615$ DMRs) compared

111 with differentially expressed genes (DEGs) between brain and kidney RNA-seq samples.

112 Currently, available RNA-seq data from koalas do not include sufficient biological replicates. We

113 overcame this limitation by simulating replicates within each RNA-seq data set (NOISeq, (24))

114 and identified 600 putative DEGs (probability of differential expression $>95 \%$ according to the

115 NOISeq). We found that DMGs were significantly more likely to be differentially expressed than

116 genes that did not contain DMRs, exhibiting a 1.54-fold enrichment $\left(\chi^{2}=33.07, \mathrm{p}<0.0001\right)$. In

117 addition, differential expression between tissues was significantly correlated with differential

118 promoter DNA methylation between tissues (Supplementary Fig. 3A). Both relative

119 hypomethylation and relative hypermethylation of gene bodies were associated with increased

120 expression (Supplementary Fig. 3B). In Fig. 1D and 1E, we show DNA methylation patterns of

121 two representative genes containing brain specific DMRs.

\section{Global hypomethylation of female $\mathbf{X}$ chromosome in koalas}

124 The koala genome project used cross-species chromosome painting data to identify 24 putative $\mathrm{X}$ 125 chromosome scaffolds and 406 putative autosomal scaffolds (25). As expected from 2:1 ratio of $126 \mathrm{X}$ chromosomes in females compared to males, the median depth of coverage of CpGs on the 127 putative X scaffolds were consistently higher (approximately 2-fold) in female samples compared 
128 to male samples ( $\mathrm{p}<2.2 \times 10^{-16}$, Mann-Whitney $U$ test, Supplementary Fig. 4A). Additionally, the

129 proportion of reads mapped to the putative $\mathrm{X}$ scaffolds showed a distinct bimodal distribution

130 whereby the male samples cluster close to $1.3 \%$ and the female samples cluster near $2.4 \%$

131 (Supplementary Fig. 4B). In contrast, male and female samples were indistinguishable with respect

132 to read mapping to putative autosomes (Supplementary Fig. 4D).

133 The global DNA methylation level of the female X chromosome was lower than that of the

134 male $X$ chromosome in all koala tissues examined (Fig. 2A, B and Supplementary Fig. 5, p $<2.2$

$135 \times 10^{-16}$, Mann-Whitney $U$ test). A comparison to autosomal DNA methylation indicated that the $\mathrm{X}$ 136 chromosome exhibits reduced DNA methylation in females. Consequently, we use the term

137 'female hypomethylation' (as opposed to male hypermethylation) (Fig. 2C) consistently in this

138 work. As a comparative basis of the DNA methylation patterns observed in eutherian mammals, 139 we performed parallel analyses on human DNA methylation data (methods). Human X 140 chromosomes were also globally hypomethylated in females compared to males (Fig. 2A, C).

141 Female hypomethylation was observed in all functional regions across the $\mathrm{X}$ chromosome (Fig. 142 2D), but most pronounced in gene bodies and intergenic regions. Promoters showed the least 143 difference between males and females. As expected, the autosomal scaffolds did not display a 144 significant variation between female and male methylation levels in any functional region (Fig. 145 2E). Fig. $2 \mathrm{~F}$ depicts the DNA methylation difference between male and female X chromosomes 146 in humans. In contrast to the pattern observed in koalas, promoters on the human female $\mathrm{X}$ 147 chromosomes were hypermethylated compared to those on the male X chromosome, congruent 148 with previous studies (26-28).

\section{Promoter DNA methylation is not a universal driver of sex-specific expression in koalas}

151 To investigate the implications of the observed sex-specific DNA methylation, we once again 152 utilized the published RNA-seq koala transcriptomes (23). Of the total RNA-seq dataset, only one 153 tissue (kidney) had expression data from both a male and female koala that could be directly 154 compared to our methylome dataset. Consequently, the other tissues were not considered for 155 further analysis. We calculated the log-transformed fold change of female and male gene 156 expression values using NOISeq, capitalizing on its ability to simulate technical replicates. Of the 157209 X-linked genes, 36 (17.2\%) exhibit female overexpression while 11 (5.3\%) show male 158 overexpression (probability of differential expression $>95 \%$ based on NOISeq, Supplementary 
159 Fig. 6A). Although, on average, autosomal genes also exhibited slight female-bias of expression

160 (Supplementary Fig. 6B, C), the increase was more substantial in the $\mathrm{X}$ chromosome (mean

161 chromosome $\mathrm{X}$ female to male $\log _{2}$ fold change $=0.50$, autosome female to male expression $\log _{2}$

162 fold change $=0.24$ ). We calculated the female and male fractional methylation difference in $\mathrm{X}$

163 chromosome-linked promoters and gene bodies correlated with gene expression difference $(\mathrm{N}=$

164209 gene bodies and $\mathrm{N}=206$ promoters, excluding 3 promoters with CpGs coverage $<3$ ). In

165 promoters, no significant relationship was observed (Supplementary Fig. 7A). In fact, the

166 proportions of significantly female-over expressed genes were similar between female-

167 hypermethylated promoters and female-hypomethylated promoters (Supplementary Fig. 7B).

168 Interestingly, female and male DNA methylation difference in gene bodies showed an overall

169 negative correlation with gene expression (Spearman's rank correlation coefficient, $\rho=-0.14, \mathrm{p}=$

170 0.04, Supplementary Fig. 7C). A deeper analysis of the relative DNA methylation and expression

171 levels revealed that both extreme hypomethylation and hypermethylation are associated with

172 increased expression (Supplementary Fig. 7C) attesting to the complexity and heterogeneity of the

173 relationship between gene body DNA methylation and gene expression.

175 The $R s x$ region displays a pattern suggesting methylation driven control of $X$ chromosome 176 regulation in koalas

177 Sex-specific DNA methylation of a key regulator of XCI in other marsupials, the $\ln c$ RNA gene

$178 R s x$, has been associated with its differential expression $(8,10)$. Based on the sequence homology

179 with the Rsx gene from the gray short-tailed opossum (Monodelphis domestica) (8), we identified

180 a $29.8 \mathrm{~Kb}$ candidate $R s x$ sequence (Methods), using PacBio long read sequencing by Johnson et

181 al. (25). Interestingly, we observed a female hypomethylated region containing two CpG islands

182 upstream of the candidate $R s x$ covering 101 CpGs exhibiting a 36\% reduction in mean female

183 DNA methylation compared to mean male DNA methylation (mean sex difference: $-0.36 \pm 0.14$,

184 Fig. 3). The mean DNA methylation difference between sexes within this region was in the top

$18523 \%$ of the distribution of differences across all X-linked scaffolds of the combined tissues. The

186 expression of the $\operatorname{lncRNA}$ annotated within the candidate $R s x$ gene region was significantly greater

187 in females (mean normalized read count $=6987.1)$ than in males (mean normalized read count $=$

$18816, \mathrm{p}<0.05$ from DeSeq2 using the Wald test). When considering a subset of tissue expression 
data with matched male and female samples (spleen, kidney, and lung), the results remained robust across different tools to measure differential gene expression (Table 3).

\section{Identification of novel candidate $X$-linked scaffolds by sex-specific methylation patterns}

193 We have demonstrated above that X-linked scaffolds exhibit several distinctive WGBS patterns in

194 koalas, including different depths of coverage per $\mathrm{CpG}$, distinctive clustering based on the 195 proportion of mapped reads, and unique methylation distributions between females and males

196 (Supplementary Fig. 4). We thus quantified DNA methylation differences between females and 197 males to determine if additional candidate $\mathrm{X}$ scaffolds existed within the $6.7 \%$ of the koala 198 assembly that remained unclassified. We identified an additional 98 scaffolds that showed a clear 199 shift towards female hypomethylation (mean female-male $5 \mathrm{mC}$ for all candidate $\mathrm{X}$ scaffolds was $200-0.25 \pm 0.12$ ) (Supplementary Tables 4,5). These candidate scaffolds contributed 14.6 Mb (21.5\%) 201 to the total genomic region annotated as the koala X chromosome. All candidate scaffolds followed 202 the expected bimodal distribution seen in the putative X scaffolds; male samples clustered around $203 \quad 0.3 \%$ and the female samples clustered around 0.5\% (Supplementary Fig. 4C). This clustering 204 pattern could be attributed to the 2:1 ratio of $\mathrm{X}$ chromosomes in females compared to males that 205 was not observed in putative autosomes.

\section{Discussion}

208 In this study, we analyzed novel nucleotide-resolution genomic DNA methylation maps of five 209 tissues of a male and a female koala. The overall DNA methylation levels of koala tissues are on 210 par with those in other mammals $(27,29,30)$, and show heavy genome-wide DNA methylation 211 punctuated by hypomethylation of $\mathrm{CpG}$ islands (e.g., Fig. 1D,E). Tissue-specific differential DNA 212 methylated regions (DMRs) were significantly enriched in gene bodies. Gene body methylation is 213 an ancestral form of DNA methylation in animal genomes (e.g., $(31,32)$ ), yet its role in regulation 214 of gene expression is less well understood than promoter DNA methylation owing in part to the

215 heterogeneity of gene body regions with respect to sequence length, composition and functionality 216 of introns and exons, and $\mathrm{CpG}$ density compared to promoter regions $(31,33)$. For example, DNA 217 methylation levels of the first exons/introns of genes are negatively correlated with gene 218 expression (34-36), and tend to be different from downstream genic regions (35). On the other 219 hand, high levels of cumulative gene body DNA methylation is positively correlated with gene 
220 expression and may reduce spurious transcription of intragenic RNA (37, 38). These studies

221 illustrate the potential for both positive and negative regulatory control of gene expression by gene 222 body DNA methylation.

223 We show that female X chromosomes are globally hypomethylated compared to both the

224 male X chromosome and the autosomes of both sexes (Fig. 2). Even though it may appear 225 counterintuitive at the first glance, the hypomethylation of female $\mathrm{X}$ chromosome appears as a 226 common feature of eutherian and marsupial mammals. Hellmann and Chess (26) showed that the 227 inactive $\mathrm{X}$ chromosomes of humans had reduced gene body DNA methylation. Recent whole 228 genome bisulfite sequencing analyses of mouse (27) and humans (28) showed that the 229 hypomethylation of inactive $\mathrm{X}$ chromosome is pervasive in gene bodies and intergenic regions 230 (Fig. 4). Waters et al. (19) performed reduced representation bisulfite sequencing (RRBS) of 231 several species including mouse and opossum, and concluded that the reduction of methylation in 232 gene bodies was specific to marsupials, but not in eutherians (19). The reason why they did not 233 observe methylation difference in mouse might be due to the inherent bias of RRBS, which 234 disproportionately samples regions with high GC content (20). High GC-content regions tend to 235 be hypomethylated $(39,40)$ and show less variation of DNA methylation. For example, the degree 236 of methylation difference between sexes in koala promoters is small compared to other genomic 237 regions, at least partly due to high GC content of promoters (Supplementary Fig. 8). How 238 chromosome-wide DNA hypomethylation is linked to chromosome-wide gene silencing is 239 currently unknown. Interestingly, marsupial genomes harbor an additional copy of DNMT1 (41), 240 which could lead to functional divergence between the mammalian lineages. Analyses of DMNT 241 expression however did not indicate significant differential expression of DNMTs between sexes 242 (probability of differential expression using NOISeq $<95 \%$ ). Additional analysis with broad and 243 balanced sampling of both sexes is necessary to rigorously test the impact of the marsupial DNMT1 244 duplication on gene expression.

245 Interestingly, DNA methylation signatures of $R s x$, the major player in XCI initiation in 246 marsupials (8), suggest that koala $R s x$ expression is regulated by promoter CpG island DNA 247 methylation (Fig. 3). Wang et al. (10) also showed differential DNA methylation of Rsx promoter 248 in opossum. Therefore, regulation of the key initiator of XCI via DNA methylation is another 249 parallel feature between eutherians and marsupials Notably, a recent study (42) found that Xist and 
Rsx harbor non-linear sequence similarity. Consequently, their shared functionality may be partially due to characteristics of tandem repeat regions.

In summary, we show that gene body DNA methylation is an important contributor to differential expression between tissues in koala. We also show that the global hypomethylation of

254 female $\mathrm{X}$ chromosome is a conserved feature of $\mathrm{X}$ chromosome regulation in eutherian and 255 marsupial mammals. However, X chromosome promoter methylation and the subsequent effect 256 on the regulation of gene expression appear to be divergent between these two lineages. 257 Regulation of the Rsx, on the other hand, is supported by DNA methylation, which mirrors the 258 regulation of the eutherian Xist locus. Together, these conclusions illuminate the intricate 259 evolutionary pathways that have diverged and converged to influence $\mathrm{X}$ chromosome regulation, $260 \mathrm{XCI}$, and dosage compensation in eutherian and marsupial mammals.

\section{Methods}

\section{Whole genome bisulfite sequencing and processing}

264 All genomic DNA was extracted using a Bioline Isolate II Genomic DNA Extraction Kit (Cat\#. 265 BIO-52067) following the recommended protocol with an additional DNAse free RNaseA $266(100 \mathrm{mg} / \mathrm{ml})($ Qiagen cat. \#19101) treatment before column purification. 20mg tissue samples from 267 brain, kidney, lung, skeletal muscle, and pancreas from a female koala, "Pacific Chocolate" 268 (Australian Museum registration M.45022), and a male koala, "Ben" (Australian Museum 269 registration M.47723), were supplied to the Ramaciotti Centre for Genomics for methylome 270 sequencing. The bisulfite conversion was carried out by using the EX DNA Methylation-Lightning 271 Kit (Zymo cat. \#D5030) and the WGBS libraries were constructed using the TruSeq DNA 272 methylation kit (Illumina cat.\# EGMK81213). The libraries were sequenced on a NovaSeq6000 273 S2 (Illumina) using the $2 \times 100 \mathrm{bp}$ PE option. Information on samples, coverage, and read counts 274 are provided in Supplementary Table 1. Processing of the WGBS data followed previous studies 275 (30). Bisulfite conversion rates were estimated for each WGBS sample following the methodology 276 of methPipe's bsrate (43) (Supplementary Table 1). Strand-specific methylation calls were 277 combined, and all samples were filtered to remove CpGs covered by fewer than three reads. 
A hierarchical clustering tree was drawn using the fractional methylation profiles of all ten samples using hclust from R's stats package. The distance matrix was calculated using Euclidean distances and the agglomeration method used was Ward's method. The data for the final tree was visualized using R's dendextend package (44). Bismark generated CpG reports were filtered to remove

284 scaffolds that were less than $2 \mathrm{Mb}$ in length, retaining $3.03 \times 10^{9}(94.8 \%)$ of the genome. DMRs

285 were called using BSmooth (22), with additional filtering of a minimum fractional methylation 286 difference of $0.3(30 \%)$ and at least $5 \mathrm{CpG}$ sites. DMRs were considered shared between tissues if 287 they overlapped by at least 50\%. Using koala gene annotations from Ensembl 288 (Phascolarctos_cinereus.phaCin_unsw_v4.1.97 release), promoters were defined as regions 289 located $1000 \mathrm{bp}$ upstream of the identified transcription start site (TSS). To test the enrichment of 290 tissue-specific DMRs in functional regions, we generated 10,000 length and GC content matched 291 controls for all unique DMRs. The lung and kidney samples shared the most similar methylation 292 profiles and consequently had few tissue-specific DMRs (Fig. 1C). Because of this feature, the 293 corresponding gene sets were combined for these two tissues. Functional annotation and GO term 294 enrichment analysis was performed utilizing the ToppGene Suite (45).

Differential DNA methylation between sexes

297 Johnson et al. (25) used cross-species chromosome painting data and linked 406 scaffolds spanning $2982.9 \mathrm{~Gb}$ of sequence data to autosomal scaffolds from chromosomes 1-7 and 24 scaffolds covering $29968 \mathrm{Mb}$ of sequence data to chromosome X, leaving $6.7 \%$ of the genome unclassified. To explore 300 the same relative amount of genomic space, we randomly sampled a subset of the autosomal 301 scaffolds that were length matched with the $\mathrm{X}$ chromosome scaffolds, which we called the 302 "matched autosome" dataset. These scaffolds were divided into 10-kb bins and the difference in 303 mean fractional methylation at each $10-\mathrm{kb}$ bin was compared between male and female samples

304 for all tissues. For the analysis of human data, we used WGBS fractional methylation reports from 305 a male brain (Epigenome ID: E071) and a female brain (Epigenome ID: E053) and the human 306 known gene annotations from Ensembl (hg19 release). Due to its similarity in size to the human $307 \mathrm{X}$ chromosome, we used data from human chromosome 8 as our representative autosome in the 308 comparative analysis. 
311 To isolate candidate X-linked scaffolds from the 1,477 unclassified koala scaffolds, we binned the

312 unclassified scaffolds into $10-\mathrm{kb}$ windows and calculated the mean fractional methylation of the

313 associated CpGs. We then determined the average female and male methylation differences across

314 the bins and plotted the density of the differences for all five tissues. SVY and DS proceeded to

315 independently select scaffolds that exhibited a statistically significant shift towards female

316 hypomethylation from zero. The scaffolds that showed significant female hypomethylation in all

317 five tissues and were selected by both SVY and DS were isolated $(n=98$ covering 14.6 Mb of

318 sequence with mean female-male $5 \mathrm{mC}=-0.25 \pm 0.12$ ). As an external validation, the percent of

319 reads mapping to the putative X-linked and autosome-linked scaffolds over the total number of

320 mapped reads was computed for the male and female sample in all tissues.

Annotation of the koala $R s x$

323 To annotate the genomic region around $R s x$, we downloaded the published genome sequence fasta

324 files for the partial assembly of $R s x$ from the gray short-tailed opossum (8) and the complete

325 PacBio assembly of the koala $R s x(25,42)$. We used BLASTN 2.2 .29 (46) to align both sequences

326 to the koala reference genome (phaCin_unsw_v4.1) and obtained genomic coordinates. The entire

327 assembled koala Rsx sequence aligned with 100\% identity and no gaps. Only one $30.4 \mathrm{~kb}$

328 transcript, a novel $l n c$ RNA, overlapped with the annotated $R s x$ region (overlap $>90 \%$ of transcript)

329 and was used to evaluate gene expression.

\section{Analysis of differential gene expression}

332 All RNA-seq expression data used in this analysis were obtained from the previously published

333 koala transcriptomes from eight females and seven males (23). To process the acquired data, we

334 followed the protocol outlined by Pertea et al. (47), using the koala GTF annotation from Ensembl

335 (Phascolarctos_cinereus.phaCin_unsw_v4.1.97.gtf.gz release) to assemble mapped reads into

336 transcripts using StringTie 2.0 (47) with the -e-b--A < gene_abund.tab> flags. We used StringTie's

337 functionality for de novo transcript assembly to identify candidate Rsx transcripts. An updated

338 GTF annotation was generated including novel transcripts using the --merge flag and then the

339 previously generated mapped reads were reassembled into transcripts guided by this GTF file.

340 DeSeq2 1.22.2 (48) was used to perform differential gene expression analysis between males and 
341 females. NOISeq 2.26.1 (24) was used for differential expression analysis due to its ability to

342 simulate technical replicates within given RNA-seq data sets when no replicates are available.

\section{Data accessibility}

345 The raw and processed methylation datasets generated in this study have been deposited and

346 accessible through GEO Series accession number GSE149600 at

347 https://www.ncbi.nlm.nih.gov/geo/query/acc.cgi?acc=GSE149600.

\section{Competing interests}

350 The authors declare that they have no competing interests.

\section{Authors' contributions}

353 D.S., D.A.P. and S.V.Y. formed the research design; D.A.P., A.G.K., D.E.A.P, and R.N.J. 354 generated the data; D.S., D.S. and S.V.Y. performed the analysis and wrote the initial draft ; all 355 authors contributed to and approved the final manuscript.

\section{Funding statement}

358 This work was supported by a grant from the National Science Foundation (MCB 1818288) and a 359 Pilot Grant from the Smooth Muscle Plasticity COBRE of the University of Nevada, Reno (funded 360 by the National Institutes of Health grant 5P30GM110767-04) to DAP, grants by the National 361 Science Foundation (MCB 1615664) and the National Institute of Health (R01MH103517) to 362 SVY. DS was partially supported by the NIH Training Grant in Computational Biology and 363 Biomedical Genomics (T32 GM105490).

\section{References}

366 1. Lyon MF. Gene Action in the X-chromosome of the Mouse (Mus musculus L.). Nature. 367 1961;190(4773):372-3.

$368 \quad 2$. Shevchenko AI, Zakharova IS, Zakian SM. The evolutionary pathway of x chromosome 369 inactivation in mammals. Acta Naturae. 2013;5(2):40-53.

3703 3. Brown CJ, Hendrich BD, Rupert JL, Lafreniere RG, Xing Y, Lawrence J, et al. The human 371 XIST gene: analysis of a $17 \mathrm{~kb}$ inactive $\mathrm{X}$-specific RNA that contains conserved repeats and is 372 highly localized within the nucleus. Cell. 1992;71(3):527-42. 
4. Heard E, Clerc P, Avner P. X-chromosome inactivation in mammals. Annu Rev Genet. 1997;31:571-610.

5. Plath K, Mlynarczyk-Evans S, Nusinow DA, Panning B. Xist RNA and the mechanism of X chromosome inactivation. Annu Rev Genet. 2002;36:233-78.

377 6. Duret L, Chureau C, Samain S, Weissenbach J, Avner P. The Xist RNA gene evolved in eutherians by pseudogenization of a protein-coding gene. Science. 2006;312(5780):1653-5. 7. $\mathrm{Ng} \mathrm{K}$, Pullirsch D, Leeb M, Wutz A. Xist and the order of silencing. EMBO Rep. $380 \quad 2007 ; 8(1): 34-9$.

$381 \quad 8$. Grant J, Mahadevaiah SK, Khil P, Sangrithi MN, Royo H, Duckworth J, et al. Rsx is a metatherian RNA with Xist-like properties in X-chromosome inactivation. Nature. 2012;487:254. 9. Sharman GB. Late DNA Replication in the Paternally Derived X Chromosome of Female Kangaroos. Nature. 1971;230(5291):231-2.

385 10. Wang X, Douglas KC, Vandeberg JL, Clark AG, Samollow PB. Chromosome-wide profiling of $\mathrm{X}$-chromosome inactivation and epigenetic states in fetal brain and placenta of the opossum, Monodelphis domestica. Genome Res. 2014;24(1):70-83.

11. Huynh KD, Lee JT. Inheritance of a pre-inactivated paternal X chromosome in early mouse embryos. Nature. 2003;426(6968):857-62. 12. Okamoto I, Otte AP, Allis CD, Reinberg D, Heard E. Epigenetic dynamics of imprinted X inactivation during early mouse development. Science. 2004;303(5658):644-9.

398 15. Ingles ED, Deakin JE. Global DNA Methylation patterns on marsupial and devil facial 13. Heard E. Delving into the diversity of facultative heterochromatin: the epigenetics of the inactive X chromosome. Current opinion in genetics \& development. 2005;15(5):482-9.

14. Koina E, Chaumeil J, Greaves IK, Tremethick DJ, Graves JA. Specific patterns of histone marks accompany $\mathrm{X}$ chromosome inactivation in a marsupial. Chromosome research : an international journal on the molecular, supramolecular and evolutionary aspects of chromosome biology. 2009; 17(1):115-26.

400 16. Rens W, Wallduck MS, Lovell FL, Ferguson-Smith MA, Ferguson-Smith AC. Epigenetic modifications on $\mathrm{X}$ chromosomes in marsupial and monotreme mammals and implications for evolution of dosage compensation. Proceedings of the National Academy of Sciences. 2010;107(41):17657.

409 19. Waters SA, Livernois AM, Patel H, O’Meally D, Craig JM, Marshall Graves JA, et al. 410 Landscape of DNA Methylation on the Marsupial X. Molecular Biology and Evolution.

412 20. Sun Z, Cunningham J, Slager S, Kocher J-P. Base resolution methylome profiling: 413 considerations in platform selection, data preprocessing and analysis. Epigenomics. 2015;7(5):813-28. content and genome size assessed from flow karyotypes: invariable low autosomal GC content. R Soc Open Sci. 2018;5(8):171539-. 
22. Hansen KD, Langmead B, Irizarry RA. BSmooth: from whole genome bisulfite sequencing reads to differentially methylated regions. Genome Biol. 2012;13(10):R83. 23. Hobbs M, Pavasovic A, King AG, Prentis PJ, Eldridge MDB, Chen Z, et al. A transcriptome resource for the koala (Phascolarctos cinereus): insights into koala retrovirus transcription and sequence diversity. BMC Genomics. 2014;15(1):786.

24. Tarazona S, Furio-Tari P, Turra D, Pietro AD, Nueda MJ, Ferrer A, et al. Data quality aware analysis of differential expression in RNA-seq with NOISeq R/Bioc package. Nucleic acids research. 2015;43(21):e140. and conservation insights from the koala genome. Nature Genetics. 2018;50(8):1102-11.

26. Hellman A, Chess A. Gene body-specific methylation on the active X chromosome. Science. 2007;315:1141-3.

430 27. Keown CL, Berletch JB, Castanon R, Nery JR, Disteche CM, Ecker JR, et al. Allelespecific non-CG DNA methylation marks domains of active chromatin in female mouse brain. Proceedings of the National Academy of Sciences. 2017;114(14):E2882. of the $\mathrm{Z}$ Chromosome between sexes in a female heterogametic system. Genome Res. 2019;29(10):1673-84.

436 29. Schultz MD, He Y, Whitaker JW, Hariharan M, Mukamel EA, Leung D, et al. Human body epigenome maps reveal noncanonical DNA methylation variation. Nature. 2015;523(7559):2126.

30. Mendizabal I, Shi L, Keller TE, Konopka G, Preuss TM, Hsieh TF, et al. Comparative Methylome Analyses Identify Epigenetic Regulatory Loci of Human Brain Evolution. Mol Biol Evol. 2016;33(11):2947-59.

31. Zemach A, McDaniel IE, Silva P, Zilberman D. Genome-wide evolutionary analysis of eukaryotic DNA methylation. Science. 2010;328(5980):916-9.

448 34. Chuang T-J, Chen F-C, Chen Y-Z. Position-dependent correlations between DNA 32. Yi SV. Birds do it, bees do it, worms and ciliates do it too: DNA methylation from unexpected corners of the tree of life. Genome Biol. 2012;13(10):174.

33. Jones PA. Functions of DNA methylation: islands, start sites, gene bodies and beyond. Nature reviews Genetics. 2012;13(7):484-92. methylation and the evolutionary rates of mammalian coding exons. Proceedings of the National Academy of Sciences. 2012;109(39):15841. the first exon is tightly linked to transcriptional silencing. PloS one. 2011;6(1):e14524. 36. Anastasiadi D, Esteve-Codina A, Piferrer F. Consistent inverse correlation between DNA methylation of the first intron and gene expression across tissues and species. Epigenetics \& Chromatin. 2018;11(1):37.

37. Neri F, Rapelli S, Krepelova A, Incarnato D, Parlato C, Basile G, et al. Intragenic DNA methylation prevents spurious transcription initiation. Nature. 2017;543(7643):72-7.

458 38. Huh I, Zeng J, Park T, Yi SV. DNA methylation and transcriptional noise. Epigenetics \& 459 chromatin. 2013;6(1):9-.

460 39. Cotton AM, Price EM, Jones MJ, Balaton BP, Kobor MS, Brown CJ. Landscape of DNA 461 methylation on the $\mathrm{X}$ chromosome reflects $\mathrm{CpG}$ density, functional chromatin state and $\mathrm{X}$ 462 chromosome inactivation. Hum Mol Genet. 2015;24(6):1528-39. 

origins exhibit contrasting patterns of regional substitution rate variation. PLoS Computational 465 Biology. 2008;4(2):e1000015.

466 41. Alvarez-Ponce D, Torres-Sanchez M, Feyertag F, Kulkarni A, Nappi T. Molecular 467 evolution of DNMT1 in vertebrates: Duplications in marsupials followed by positive selection. 468 PloS one. 2018;13(4).

469 42. Sprague D, Waters SA, Kirk JM, Wang JR, Samollow PB, Waters PD, et al. Nonlinear 470 sequence similarity between the Xist and Rsx long noncoding RNAs suggests shared functions of tandem repeat domains. RNA. 2019;25(8):1004-19.

473 Database and Analysis Pipeline to Facilitate Integrative and Comparative Epigenomics. PLOS 474 ONE. 2013;8(12):e81148.

475 44. Galili T. dendextend: an $\mathrm{R}$ package for visualizing, adjusting and comparing trees of 476 hierarchical clustering. Bioinformatics. 2015;31(22):3718-20.

477 45. Chen J, Bardes EE, Aronow BJ, Jegga AG. ToppGene Suite for gene list enrichment 478 analysis and candidate gene prioritization. Nucleic acids research. 2009;37(Web Server 479 issue):W305-11.

480 46. Zhang Z, Schwartz S, Wagner L, Miller W. A greedy algorithm for aligning DNA 481 sequences. Journal of computational biology : a journal of computational molecular cell biology. 482 2000;7(1-2):203-14.

483 47. Pertea M, Kim D, Pertea GM, Leek JT, Salzberg SL. Transcript-level expression analysis 484 of RNA-seq experiments with HISAT, StringTie and Ballgown. Nature Protocols. 485 2016;11(9):1650-67.

486 48. Love MI, Huber W, Anders S. Moderated estimation of fold change and dispersion for 487 RNA-seq data with DESeq2. Genome Biol. 2014;15(12):550.

488 49. Ogawa Y, Kakumoto K, Yoshida T, Kuwako K-i, Miyazaki T, Yamaguchi J, et al. Elavl3 489 is essential for the maintenance of Purkinje neuron axons. Scientific Reports. 2018;8(1):2722. 


\section{Figure Legends}

493 Figure 1. Overview of DNA methylation patterns across the koala genome. (A) Hierarchical 494 clustering of DNA methylation of five tissues. (B) Tissue-specific and shared differentially 495 methylation regions (DMRs) between tissues. (C) Enrichments of DMRs in each functional region 496 in comparison to length and GC matched control regions $(* * * p<0.0001$, n.s. Not significant, from 497 10,000 bootstraps) from brain samples. Error bars depict standard deviation. (D-E) Examples of 498 two brain-specific DMRs (highlighted in grey) with corresponding CpG fractional methylation for 499 the two brain (red) and eight remaining tissues (blue). Line smoothing performed using local 500 regression (LOESS). (D) ELAVL3 is a neural specific RNA-binding protein linked to the 501 maintenance of Purkinje neuron axons (49). A $1.84 \mathrm{~kb}$ region containing three CpG Islands (CGI) 502 and overlapping the first exon of ELAVL3 was hypermethylated in the brain compared to all other 503 tissues, and this gene was up-regulated in the brain compared to the kidneys (probability of 504 differential expression $>98 \%$ from NOISeq). (D) $A L D O C$ encodes a catalytic enzyme responsible 505 for the conversion of fructose-1,6-bisphosphate to glyceraldehyde 3-phosphate and 506 dihydroxyacetone phosphate. A 945 bp, brain-specific hypomethylated DMR overlaps the 507 promoter and part of $A L D O C$ 's gene body. This gene was up-regulated in brain samples compared 508 to kidney samples (probability of differential expression $>96 \%$ from NOISeq).

Figure 2. Global patterns of female and male DNA methylation $(5 \mathrm{mC})$ in eutherian and marsupial X chromosomes. (A) Comparison of chromosome-wide DNA methylation between 512 sexes in human and koala brains. (B) Distributions of the DNA methylation difference between 513 female and male koalas in autosomes and the $\mathrm{X}$ chromosome. (C) Comparing fractional DNA 514 methylation between males and females demonstrate that the female $\mathrm{X}$ chromosomes are 515 hypomethylated in both humans and koalas. (D-F) DNA methylation differences between males 516 and females of (D) the X chromosome and (E) autosomes of koalas, and (F) the X chromosome of

517 humans for all male (purple) and female (orange) samples. Line smoothing was performed using 518 local regression (LOESS). (G) Average fractional methylation of CpGs in 100-bp sliding windows 519 using a $10 \mathrm{bp}$ step size in a $5 \mathrm{~Kb}$ region upstream and downstream of all chromosome $\mathrm{X}$ linked 520 gene's transcription start sites (TSSs) across koala tissues. 
522 Figure 3. Annotation of genomic of DNA methylation (5mC) around $R s x$. The top panel

523 identifies $\mathrm{CpG}$ islands (CGI), the middle panel reports the absolute male (purple) and female

524 (orange) fractional methylation at each $\mathrm{CpG}$, and the bottom panel shows the female and male

525 fractional methylation difference. Highlighted in grey across all panels is the female

526 hypomethylated region upstream of the Rsx TSS.

528 Figure 4. Model of DNA methylation $(5 \mathrm{mC})$ patterns across genomic functional regions for

529 eutherian mammals and marsupials. Eutherian mammals exhibit increased DNA methylation

530 of promoter regions and $\mathrm{CpG}$ islands coupled with a relative depletion of DNA methylation across

531 gene bodies and intergenic regions on the inactive $\mathrm{X}$ chromosome $\left(\mathrm{X}_{\mathrm{I}}\right)$ compared to the active $\mathrm{X}$

532 chromosome $\left(\mathrm{X}_{\mathrm{A}}\right)$ in females. Marsupial mammals share similar DNA methylation depletion in

533 gene bodies and intergenic regions of the inactive $\mathrm{X}$ chromosome; however, they diverge from

534 eutherian mammals in their promoter methylation patterns. Marsupial promoters are modestly

535 hypomethylated in the female $\mathrm{X}$ chromosomes $\left(\mathrm{X}_{\mathrm{A}}\right.$ and $\left.\mathrm{X}_{\mathrm{I}}\right)$ compared to the male $\mathrm{X}$ chromosome $536\left(\mathrm{X}_{\mathrm{M}}\right)$. 


\section{Tables}

Table 1. Functional annotation of enriched biological processes associated with gene sets containing tissue-specific differentially methylated regions (DMRs).

\begin{tabular}{llllc}
\hline \multicolumn{1}{c}{ Tissue } & \multicolumn{1}{c}{ GO biological process term } & Accession ID & p-value & q-value \\
\hline Brain & central nervous system development & GO:0007417 & $5.79 \times 10^{-13}$ & $2.71 \times 10^{-09}$ \\
Brain & generation of neurons & GO:0048699 & $1.47 \times 10^{-12}$ & $3.45 \times 10^{-09}$ \\
Brain & head development & GO:0060322 & $2.84 \times 10^{-12}$ & $3.78 \times 10^{-09}$ \\
Brain & Neurogenesis & GO:0022008 & $3.22 \times 10^{-12}$ & $3.78 \times 10^{-09}$ \\
Brain & brain development & GO:0007420 & $4.98 \times 10^{-12}$ & $4.67 \times 10^{-09}$ \\
Pancreas & response to endoplasmic reticulum stress & GO:0034976 & $2.14 \times 10^{-11}$ & $1.51 \times 10^{-07}$ \\
Pancreas & oxoacid metabolic process & GO:0043436 & $1.58 \times 10^{-10}$ & $4.04 \times 10^{-07}$ \\
Pancreas & organic acid metabolic process & GO:0006082 & $1.72 \times 10^{-10}$ & $4.04 \times 10^{-07}$ \\
Pancreas & response to endogenous stimulus & GO:0009719 & $6.34 \times 10^{-09}$ & $1.12 \times 10^{-05}$ \\
Pancreas & carboxylic acid metabolic process & GO:0019752 & $2.29 \times 10^{-08}$ & $3.24 \times 10^{-05}$ \\
Skeletal Muscle & actin filament-based process & GO:0030029 & $1.26 \times 10^{-09}$ & $5.78 \times 10^{-06}$ \\
Skeletal Muscle & actin cytoskeleton organization & GO:0030036 & $5.04 \times 10^{-09}$ & $1.16 \times 10^{-05}$ \\
Skeletal Muscle & cytoskeleton organization & GO:0007010 & $1.50 \times 10^{-08}$ & $2.30 \times 10^{-05}$ \\
Skeletal Muscle & cellular carbohydrate metabolic process & GO:0044262 & $2.19 \times 10^{-08}$ & $2.52 \times 10^{-05}$ \\
Skeletal Muscle & embryonic morphogenesis & GO:0048598 & $2.37 \times 10^{-07}$ & $2.17 \times 10^{-04}$ \\
Lung and Kidney & embryonic skeletal system morphogenesis & GO:0048704 & $1.50 \times 10^{-10}$ & $3.52 \times 10^{-07}$ \\
Lung and Kidney & embryonic organ morphogenesis & GO:0048562 & $1.61 \times 10^{-10}$ & $3.52 \times 10^{-07}$ \\
Lung and Kidney & embryonic skeletal system development & GO:0048706 & $5.24 \times 10^{-10}$ & $7.65 \times 10^{-07}$ \\
Lung and Kidney & embryonic organ development & GO:0048568 & $1.13 \times 10^{-08}$ & $1.23 \times 10^{-05}$ \\
Lung and Kidney & pattern specification process & GO:0007389 & $3.31 \times 10^{-08}$ & $2.90 \times 10^{-05}$ \\
\hline
\end{tabular}

Gene ontology (GO) terms are presented for the top five most significantly enriched results of each tissue after correcting for multiple testing (FDR < 0.05). As the numbers of tissue-specific DMRs for lung $(n=22)$ and kidney ( $n=119)$ samples were so few, the corresponding gene sets were combined for this analysis. 
Table 2. Correlation analysis of mean promoter and gene body DNA methylation and ranked gene expression.

\begin{tabular}{lccc}
\hline \multicolumn{1}{c}{ Tissue } & Genomic Region & Gene Count & Rho (p-value) \\
\hline Brain & Promoter & 5,396 & $-0.08\left(\mathrm{p}=2.28 \times 10^{-9}\right)$ \\
& Gene body & 5,443 & $-0.16\left(\mathrm{p}<2.2 \times 10^{-16}\right)$ \\
\hline Kidney & Promoter & 9,268 & $-0.12\left(\mathrm{p}<2.2 \times 10^{-16}\right)$ \\
& Gene body & 9,379 & $-0.12\left(\mathrm{p}<2.2 \times 10^{-16}\right)$ \\
\hline Lung & Promoter & 9,192 & $-0.13\left(\mathrm{p}<2.2 \times 10^{-16}\right)$ \\
& Gene body & 9,265 & $-0.19\left(\mathrm{p}<2.2 \times 10^{-16}\right)$ \\
\hline
\end{tabular}

Spearman's rank correlation coefficients $(\rho)$ and associated significances are reported for all tissues with both whole genome bisulfite sequencing (WGBS) data and RNA-seq expression data.

Table 3. Sex-based differential expression of the $\operatorname{lncRNA} R s x$ utilizing different data subsets and expression quantification tools.

\begin{tabular}{lcccc}
\hline Expression Dataset & Tool & Female Count & Male Count & Significance \\
\hline All Data $(\mathrm{n}=15)$ & DeSeq2 & 6987.1 & 16 & p-value $=0.05$ \\
Matched Data $(\mathrm{n}=6)$ & DeSeq2 & 6837.6 & 0 & $\mathrm{p}$-value $=2.04 \times 10^{-30}$ \\
Matched Data $(\mathrm{n}=6)$ & NOISeq & 7872.4 & 0.67 & Probability $=99.99 \%$ \\
Kidney Data $(\mathrm{n}=2)$ & NOISeq & 4074.4 & 0.68 & Probability $=99.99 \%$ \\
\hline
\end{tabular}

Normalized expression count values and significance of sex-based differential expression is shown for three data subsets using two expression quantification tools. All data refers to the dataset considering all 15 RNA-seq samples ( 7 male and 8 female). Matched data includes the tissues with both male and female RNA-seq samples (brain, kidney, and lung), and the kidney data is reported independently. DeSeq2 reports significance as an associated p-value from the Wald test while NOISeq reports a probability of differential expression threshold. 
bioRxiv preprint doi: https://doi.org/10.1101/2020.08.26.269068; this version posted September 11, 2020. The copyright holder for this preprint (which was not certified by peer review) is the author/funder, who has granted bioRxiv a license to display the preprint in perpetuity. It is made available under aCC-BY-NC-ND 4.0 International license.

\section{Figures}

\section{Figure 1.}

A

D

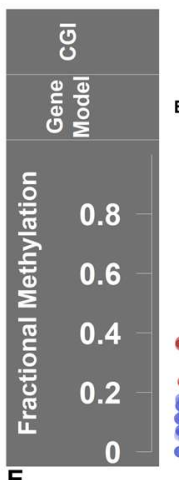

E
B

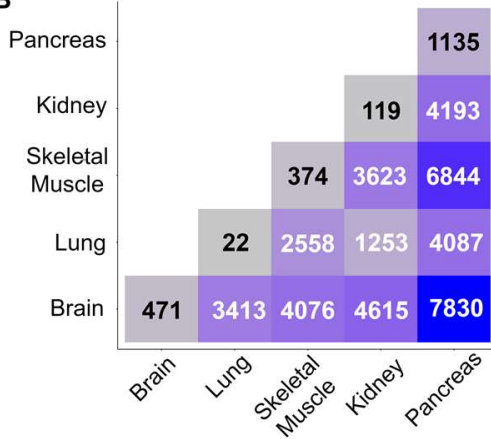

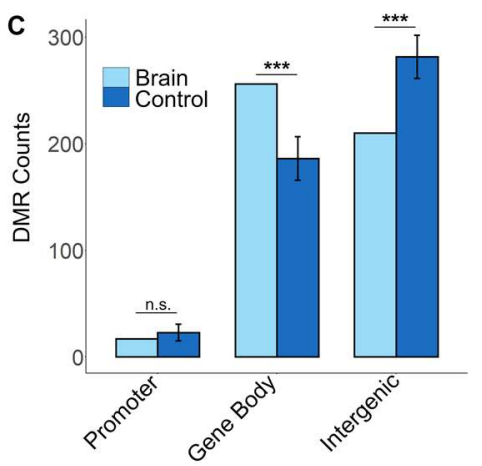

Pancreas Brain Skeletal

Muscle
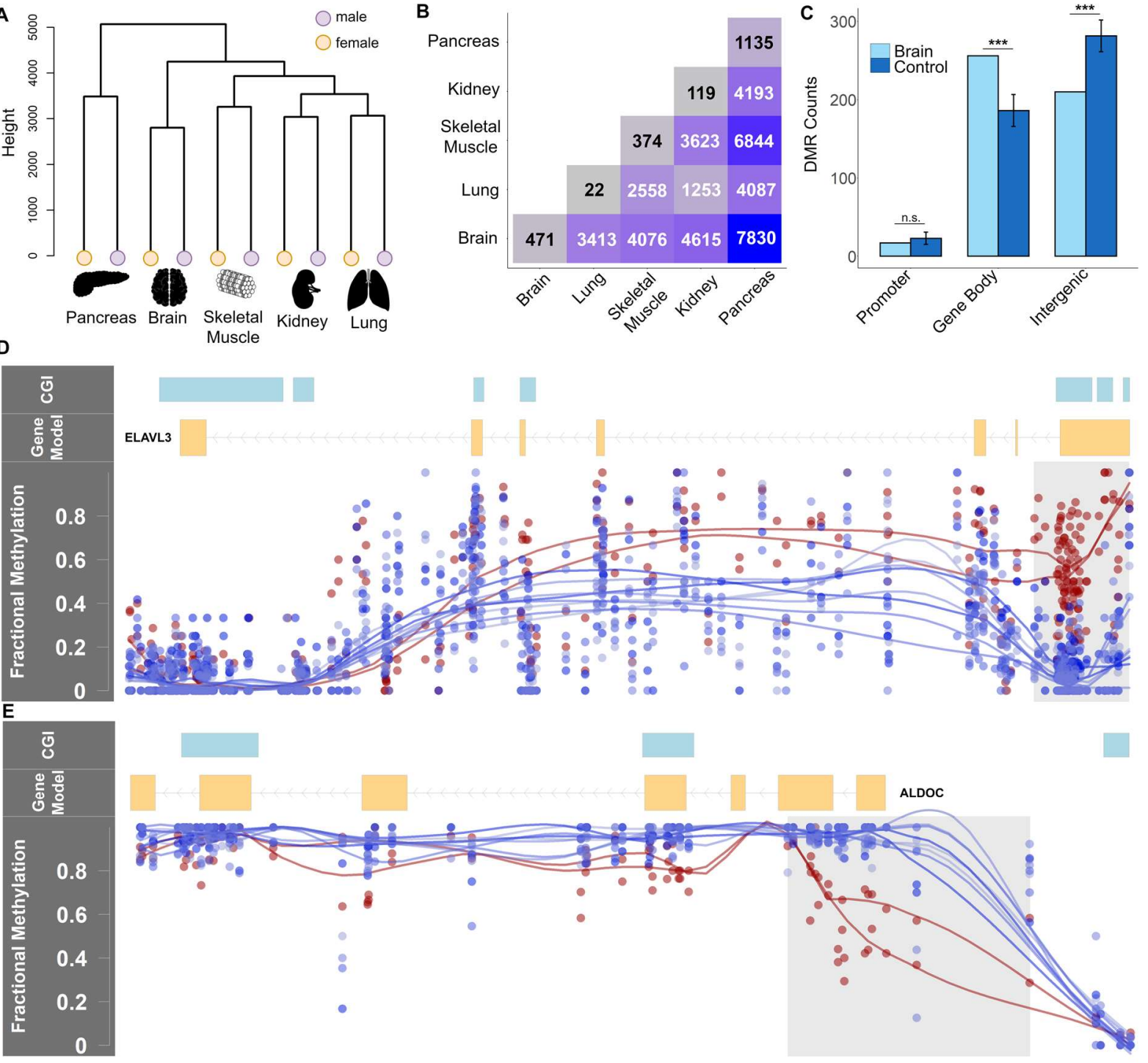
bioRxiv preprint doi: https://doi.org/10.1101/2020.08.26.269068; this version posted September 11, 2020. The copyright holder for this preprint (which was not certified by peer review) is the author/funder, who has granted bioRxiv a license to display the preprint in perpetuity. It is made available under aCC-BY-NC-ND 4.0 International license.

\section{Figure 2.}

A
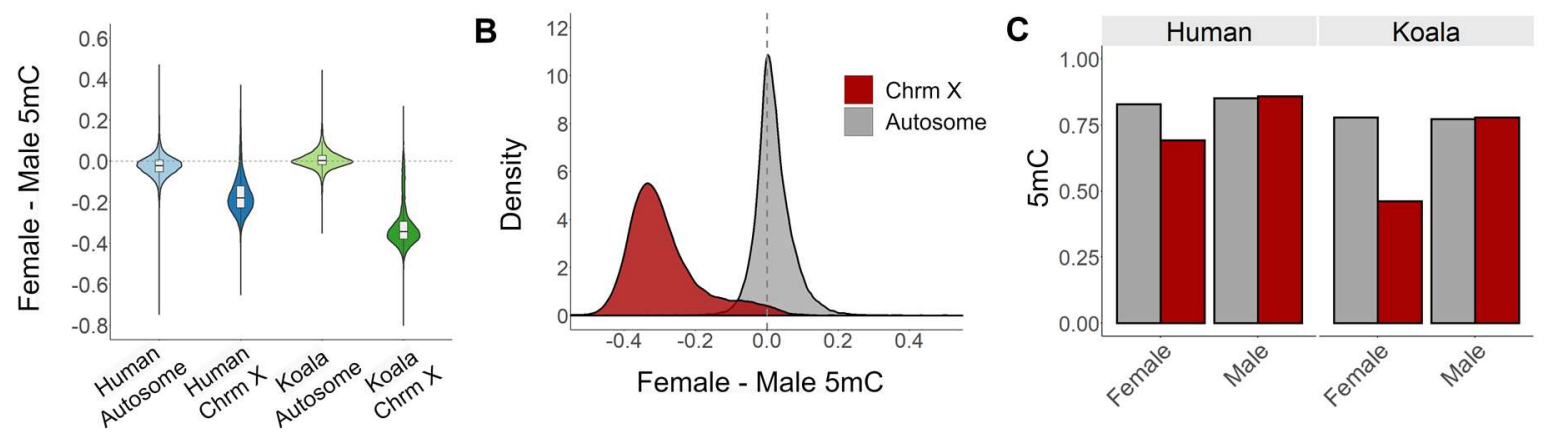

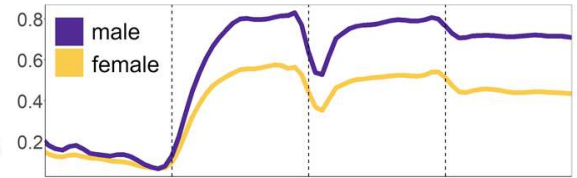

E
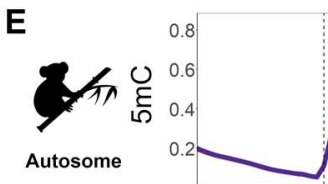

F

s

Chromosome $X$
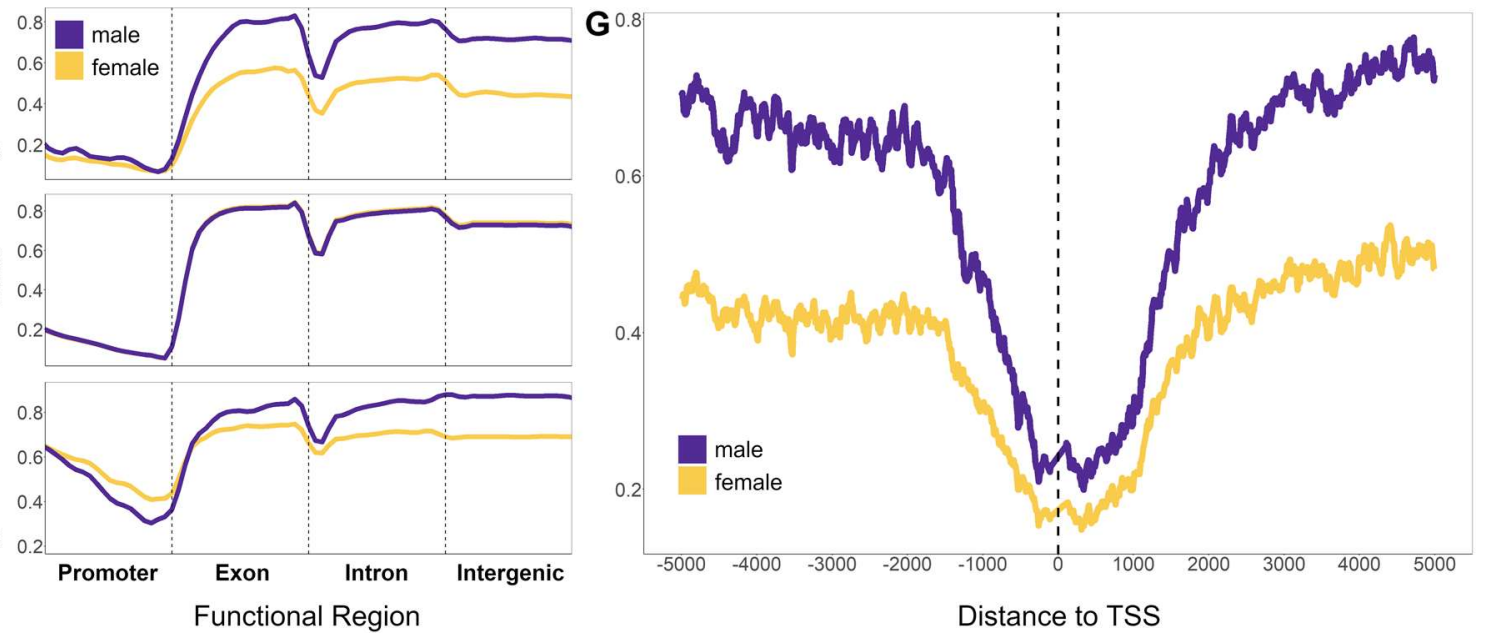
bioRxiv preprint doi: https://doi.org/10.1101/2020.08.26.269068; this version posted September 11, 2020. The copyright holder for this preprint (which was not certified by peer review) is the author/funder, who has granted bioRxiv a license to display the preprint in perpetuity. It is made available under aCC-BY-NC-ND 4.0 International license.

Figure 3.

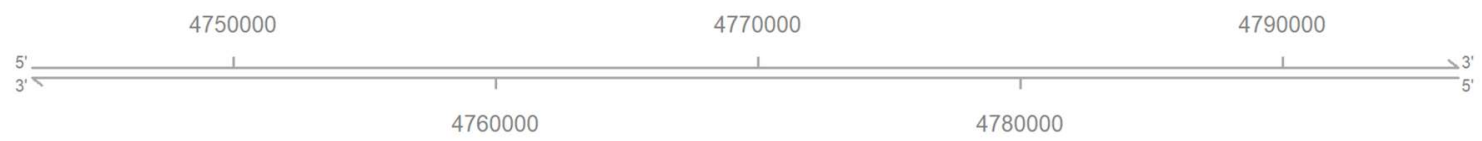

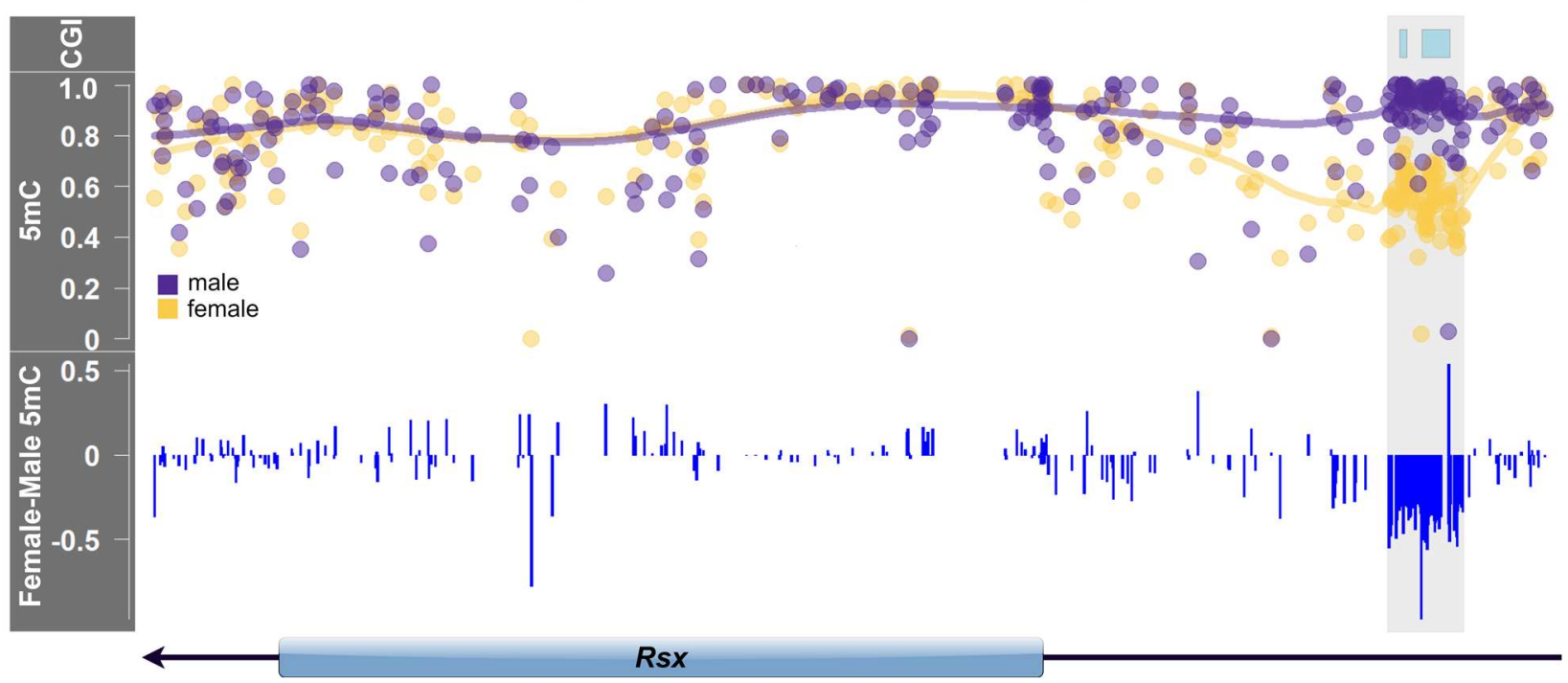


bioRxiv preprint doi: https://doi.org/10.1101/2020.08.26.269068; this version posted September 11, 2020. The copyright holder for this preprint (which was not certified by peer review) is the author/funder, who has granted bioRxiv a license to display the preprint in perpetuity. It is made available under aCC-BY-NC-ND 4.0 International license.

\section{Figure 4.}

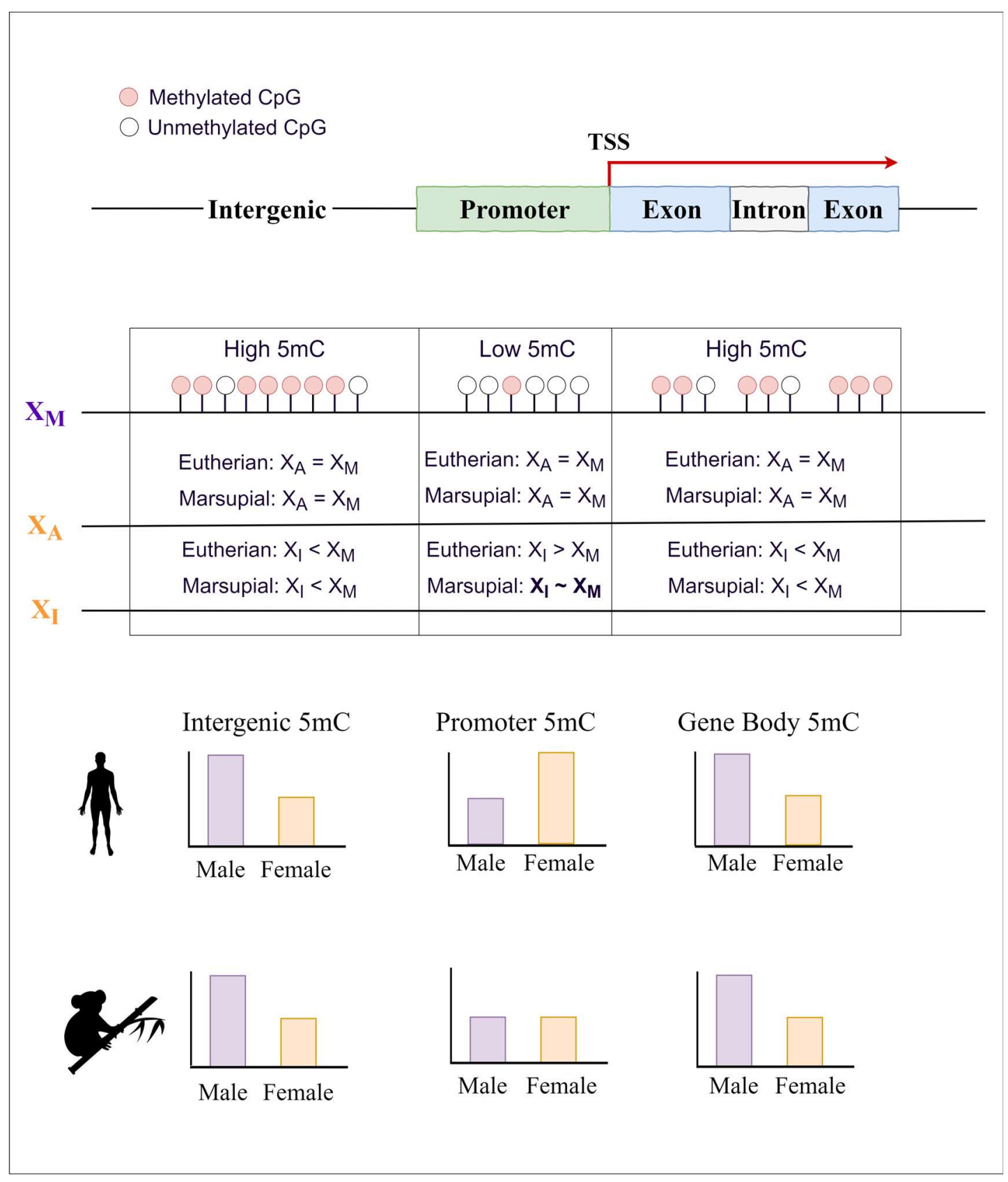




\section{Supplementary Tables}

Supplementary Table 1. Overview of whole genome bisulfite sequencing (WGBS) data for all 10 koala samples.

\begin{tabular}{|c|c|c|c|c|c|c|c|c|c|c|}
\hline Sample & $\begin{array}{l}\text { Australian } \\
\text { Museum } \\
\text { registration }\end{array}$ & Name & Tissue & $\begin{array}{c}\text { Mapped } \\
\text { Reads }\end{array}$ & $\begin{array}{c}\text { De- } \\
\text { duplicated } \\
\text { Reads }\end{array}$ & $\begin{array}{l}\text { Total } \\
\text { CpGs }\end{array}$ & $\begin{array}{c}\text { Coverage } \\
\text { (\%) }\end{array}$ & $\begin{array}{l}\text { Mean } \\
\text { Depth }\end{array}$ & $\begin{array}{c}\% \\
\text { Reads } \\
\text { Cov }> \\
3 \times \\
\end{array}$ & $\begin{array}{c}\text { Bisulfite } \\
\text { Conversion } \\
\text { Rate }\end{array}$ \\
\hline WGM145_01_S1 & M.45022 & $\begin{array}{l}\text { Pacific } \\
\text { Chocolate }\end{array}$ & Brain & 602055711 & 228244836 & 16761785 & 97.5 & 13.99 & 93.4 & 98.2 \\
\hline WGM145_04_S2 & M.47723 & Ben & Brain & 677593548 & 233893124 & 16761785 & 97.6 & 14.61 & 93.8 & 98.0 \\
\hline WGM145_06_S3 & M.45022 & $\begin{array}{l}\text { Pacific } \\
\text { Chocolate }\end{array}$ & Kidney & 559911229 & 213274030 & 16761785 & 97.2 & 13.62 & 92.5 & 98.7 \\
\hline WGM145_08_S4 & M.47723 & Ben & Kidney & 589121434 & 202094771 & 16761785 & 97.2 & 12.93 & 92.3 & 98.7 \\
\hline WGM145_09_S5 & M.45022 & $\begin{array}{l}\text { Pacific } \\
\text { Chocolate }\end{array}$ & Lung & 637878987 & 210002505 & 16761785 & 97.5 & 13.8 & 93.2 & 98.6 \\
\hline WGM145_12_S6 & M.47723 & Ben & Lung & 663204935 & 198555030 & 16761785 & 97.3 & 12.54 & 92.4 & 98.6 \\
\hline WGM145_14_S7 & M.45022 & $\begin{array}{l}\text { Pacific } \\
\text { Chocolate }\end{array}$ & $\begin{array}{l}\text { Skeletal } \\
\text { Muscle }\end{array}$ & 592423486 & 168450992 & 16761785 & 96.7 & 10.93 & 90.0 & 98.7 \\
\hline WGM145_16_S8 & M.47723 & Ben & $\begin{array}{l}\text { Skeletal } \\
\text { Muscle }\end{array}$ & 605979530 & 168220022 & 16761785 & 96.8 & 11.12 & 90.4 & 98.6 \\
\hline WGM145_19_S9 & M.45022 & $\begin{array}{l}\text { Pacific } \\
\text { Chocolate }\end{array}$ & Pancreas & 563288200 & 159866998 & 16761785 & 96.2 & 9.857 & 88.0 & 98.6 \\
\hline WGM145_20_S10 & M.47723 & Ben & Pancreas & 598508860 & 166573663 & 16761785 & 96.6 & 10.56 & 89.4 & 98.7 \\
\hline
\end{tabular}


Supplementary Table 2. GC-content for all scaffolds linked to annotated autosomes (chromosomes 1-7) and the $\mathrm{X}$ chromosome.

\begin{tabular}{ccc}
\hline Chromosome & Size $\mathbf{( M b )}$ & Total GC Content $(\mathbf{\%})$ \\
\hline 1 & 730.67 & 38.72 \\
2 & 595.75 & 38.58 \\
3 & 480.35 & 39.35 \\
4 & 412.55 & 39.38 \\
5 & 299.29 & 39.56 \\
6 & 248.35 & 38.66 \\
7 & 250.28 & 39.20 \\
$X$ & 67.91 & 42.02 \\
\hline
\end{tabular}

Supplementary Table 3. Enrichment and significance of all tissue specific DMRs compared to length and GC matched control regions.

\begin{tabular}{lllcc}
\hline Tissue & Genomic Region & DMR Counts (\%) & Enrichment & p-value \\
\hline Pancreas & Promoter & $52(4.5 \%)$ & 1.13 & 0.74 \\
& Gene body & $612(52.5 \%)$ & 1.44 & $<\mathbf{0 . 0 0 0 1}$ \\
& Intergenic & $502(43.0 \%)$ & -1.40 & $<\mathbf{0 . 0 0 0 1}$ \\
\hline Brain & Promoter & $17(3.5 \%)$ & -1.35 & 0.25 \\
& Gene body & $256(53.0 \%)$ & 1.38 & $<\mathbf{0 . 0 0 0 1}$ \\
& Intergenic & $210(43.5 \%)$ & -1.34 & $<\mathbf{0 . 0 0 0 1}$ \\
\hline \multirow{2}{*}{ Skeletal Muscle } & Promoter & $19(4.9 \%)$ & -1.23 & 0.32 \\
& Gene body & $202(51.8 \%)$ & 1.27 & $<\mathbf{0 . 0 0 0 1}$ \\
& Intergenic & $169(43.3 \%)$ & -1.26 & $\mathbf{0 . 0 0 1}$ \\
\hline \multirow{2}{*}{ Kidney } & Promoter & $5(4.0 \%)$ & -1.34 & 0.40 \\
& Gene body & $62(50.0 \%)$ & 1.25 & $<\mathbf{0 . 0 0 0 1}$ \\
& Intergenic & $57(46.0 \%)$ & -1.21 & $\mathbf{0 . 0 4}$ \\
\hline \multirow{2}{*}{ Lung } & Promoter & $1(4.3 \%)$ & -1.61 & 0.51 \\
& Gene body & $12(52.2 \%)$ & 1.37 & $<\mathbf{0 . 0 0 0 1}$ \\
& Intergenic & $10(43.5 \%)$ & -1.31 & 0.14 \\
\hline
\end{tabular}

Reported are the total counts of tissue specific differentially methylated regions (DMRs) falling within one of three annotated genomic regions: promoters, gene bodies, and intergenic regions. The enrichment of DMRs in each functional region is shown through a fold change comparison with a control dataset generated from 10,000 bootstraps using length and GC matched control regions. All significant $\mathrm{p}$-values $(\mathrm{p}<0.05)$ are highlighted in bold. 
Supplementary Table 4. Mean and median sex-based DNA methylation difference calculated for all candidate $\mathrm{X}$-scaffolds $(\mathrm{n}=98)$ by tissue.

\begin{tabular}{lcc}
\hline \multicolumn{1}{c}{ Tissue } & Median Male-Female 5mC & Mean Male-Female 5mC \\
\hline Pancreas & $-0.2282 \pm 0.15$ & $-0.2066 \pm 0.12$ \\
Brain & $-0.3273 \pm 0.14$ & $-0.2911 \pm 0.12$ \\
Skeletal Muscle & $-0.2748 \pm 0.15$ & $-0.2431 \pm 0.12$ \\
Kidney & $-0.2850 \pm 0.15$ & $-0.2567 \pm 0.12$ \\
Lung & $-0.2706 \pm 0.14$ & $-0.2437 \pm 0.11$ \\
\hline Combined Tissues & $-0.2773 \pm 0.15$ & $-0.2484 \pm 0.12$ \\
\hline
\end{tabular}

The female and male mean fractional DNA methylation (methylated reads/total reads per $\mathrm{CpG}$ ) was calculated for all $\mathrm{CpGs}$ within $10 \mathrm{~kb}$ bins across candidate scaffold. 


\section{Supplementary Figures}

Supplementary Figure 1. Enrichment of tissue specific differentially methylated regions (DMRs) falling within genomic functional regions.
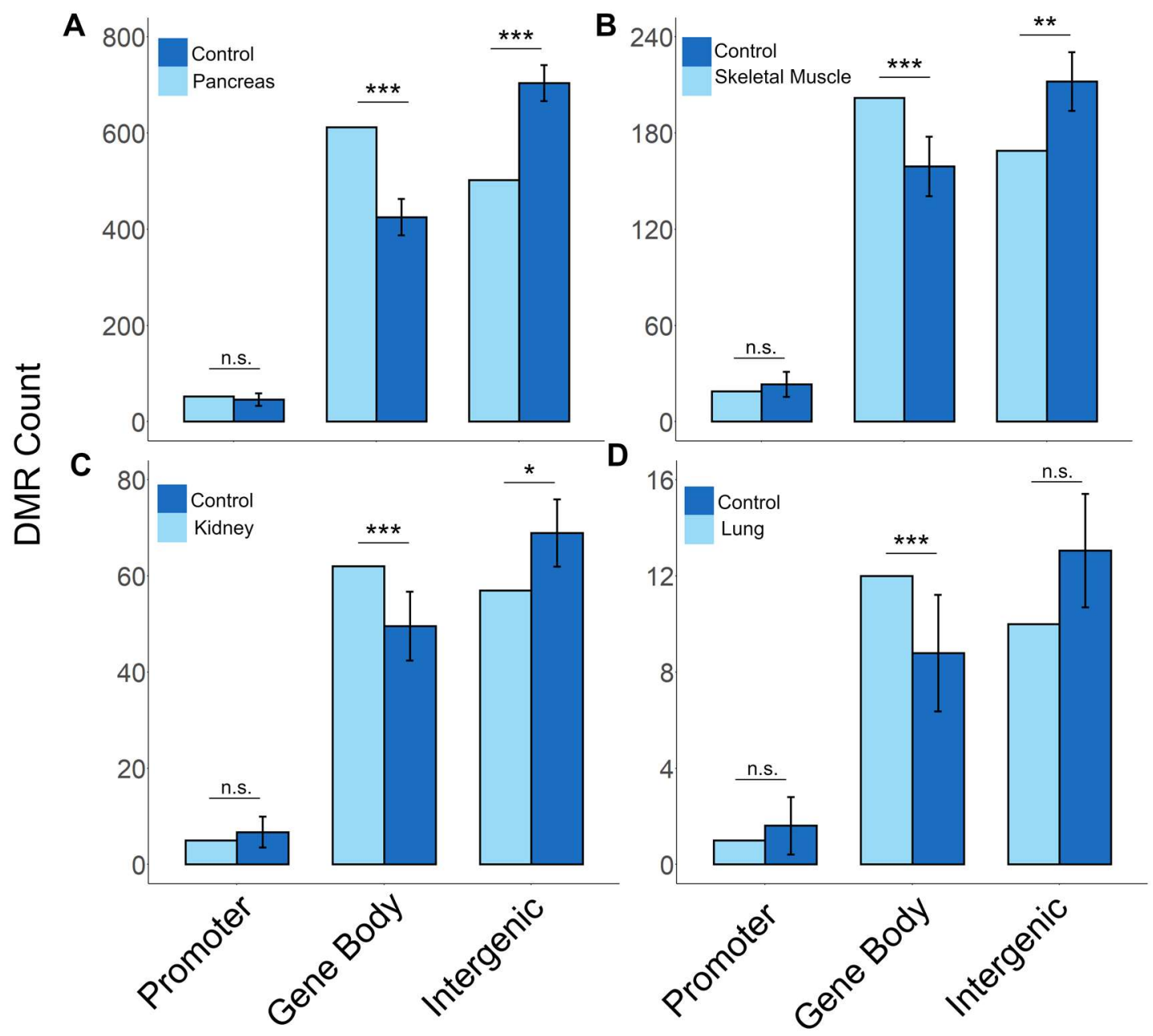

Data shown for (A) pancreas, (B) skeletal muscle, (C) kidney, and (D) lung. The enrichment of DMRs in each functional region (promoter, gene body, and intergenic regions) is shown through a comparison with length and GC matched control regions ( $* * *$ indicates $\mathrm{p}<0.0001$, ** indicates $\mathrm{p}<0.001$, * indicates $\mathrm{p}<0.05$, and non-significance is shown by n.s. based on 10,000 bootstraps). Error bars indicate standard deviation. 
Supplementary Figure 2. Correlation of gene expression and DNA methylation $(5 \mathrm{mC})$ in CpGs across promoters and gene bodies.
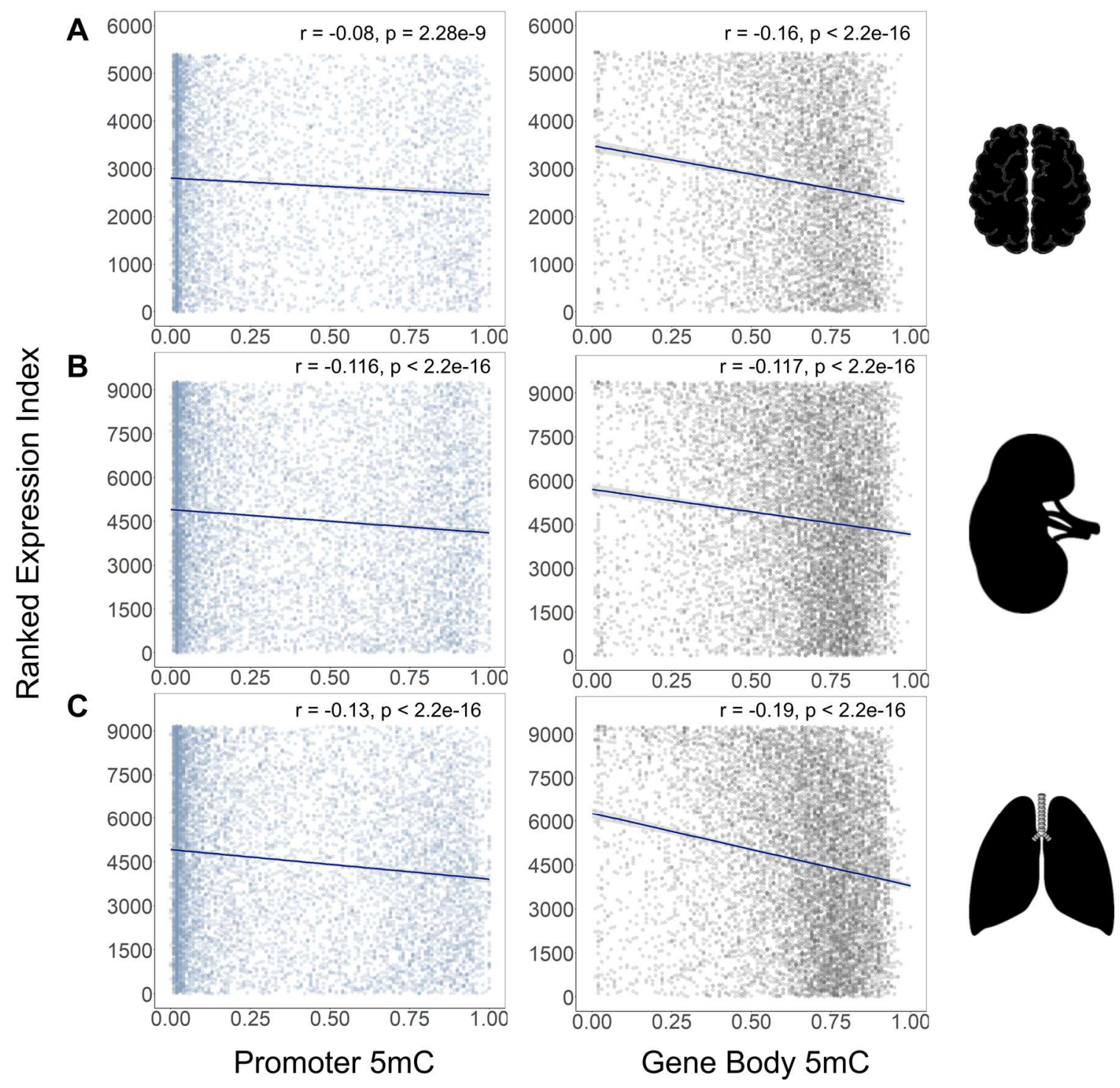

Three tissues with both whole genome bisulfite sequencing (WGBS) DNA methylation data and RNA-seq gene expression data are shown, (A) brain $(n=5,396$ promoters and $n=5,443$ gene bodies), (B) kidney ( $n=9,268$ promoters and $n=9,379$ gene bodies), and (C) lung ( $n=9,192$ promoters and $n=9,265$ gene bodies). For A-C, TPM expression values were ranked from low to high for each gene and correlated with mean fractional DNA methylation (methylated reads/total reads per CpG site). Spearman's rank correlation coefficients and the associated p-values are reported. 
Supplementary Figure 3. Correlation of tissue dependent DNA methylation $(5 \mathrm{mC})$ and gene expression from brain and kidney samples.
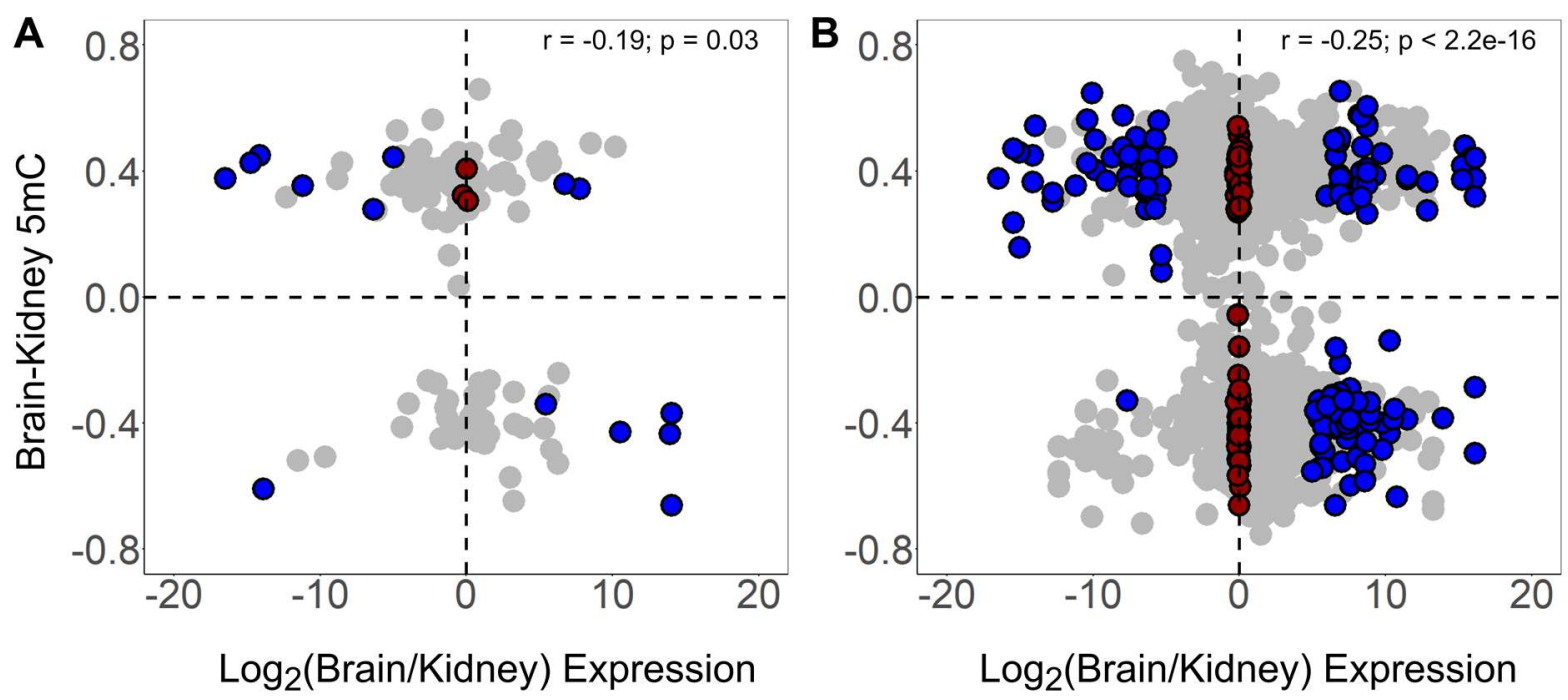

(A) The mean brain and kidney DNA methylation difference calculated for all CpGs across each gene promoter matched with the corresponding log-transformed ratio of brain to kidney expression. (B) The mean brain and kidney DNA methylation difference calculated for all CpGs across each gene body and matched with corresponding log-transformed ratio of brain to kidney expression. For A and B, Spearman's rank correlation coefficient and the associated p-value is reported. Blue dots indicate genes that are significantly differentially express between brain and kidney samples (probability of differential expression $>95 \%$ based on NOISeq) and red dots show all genes that are significantly similarly methylated in brain and kidney samples (probability of differential expression $<5 \%$ based on NOISeq). 
Supplementary Figure 4. Sex-specific CpG depth of coverage and read mapping to autosomes and $\mathrm{X}$ chromosomes.
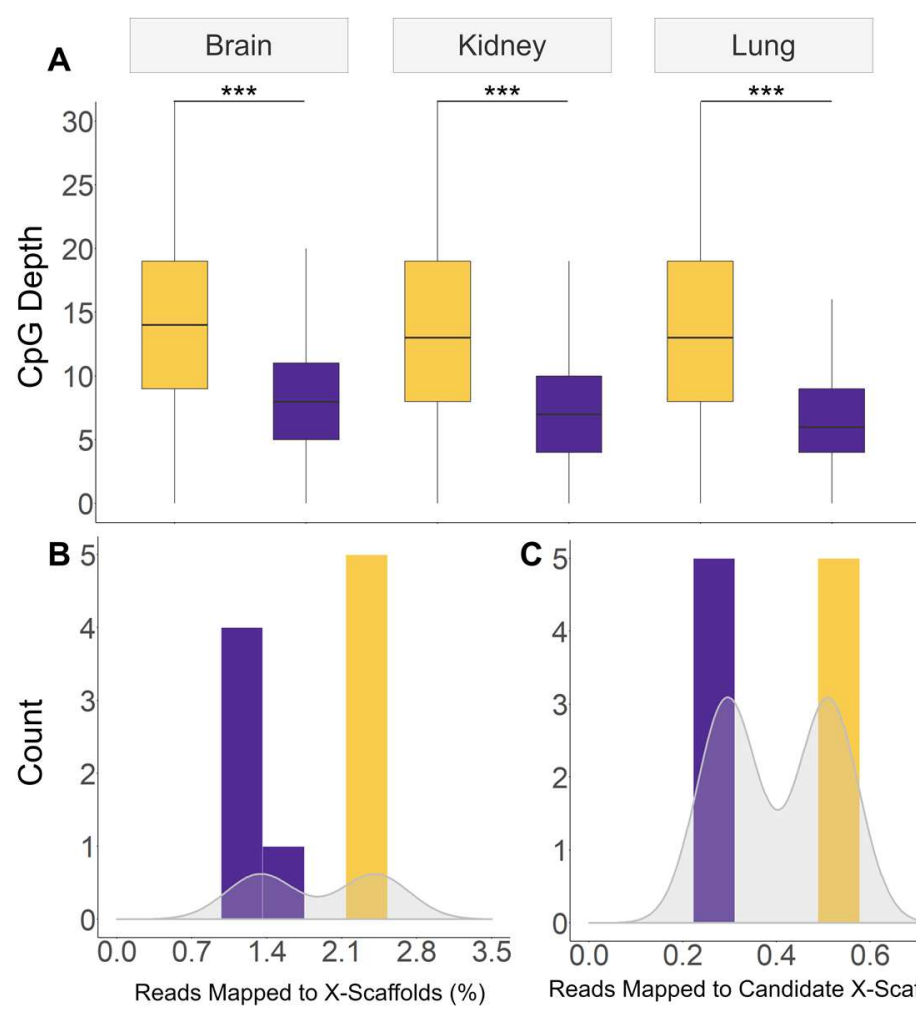

Skeletal Muscle

Pancreas
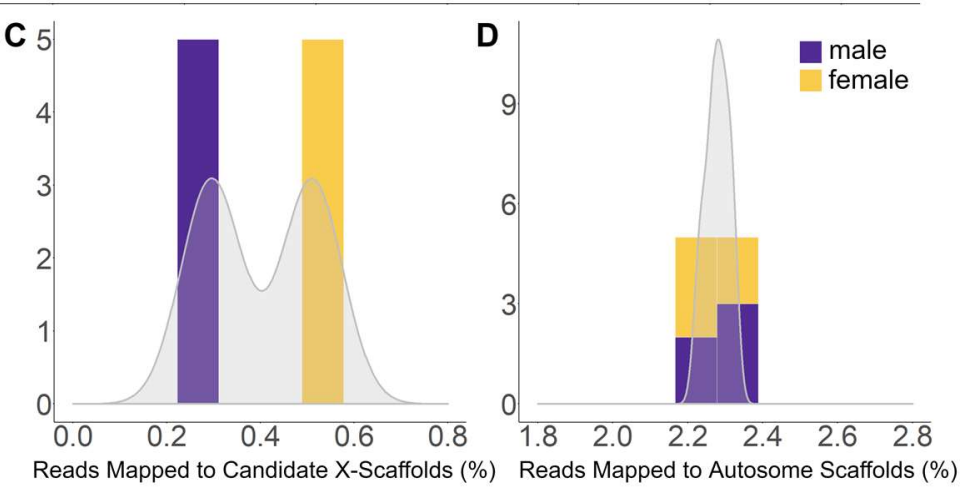

(A) For each of the five tissues, box-and-whisker plots of $\mathrm{CpG}$ depths across the $\mathrm{X}$ chromosome in male (purple) and female (orange) samples (*** indicates $\mathrm{p}<2.2 \mathrm{x} 10-16$, Mann-Whitney $\mathrm{U}$ test). Histogram and distribution of sex-based read mapping per sample $(n=10)$ to (B) X-linked scaffolds, (C) candidate X-linked scaffolds, and (D) a subset of autosome-linked scaffolds matched in length with all known X-linked scaffolds. For A-C, the percent of reads mapping to the scaffold category of interested over the total number of mapped reads in the genome was calculated for all male $(n=5)$ and female samples $(n=5)$. The known $X$-linked and candidate $X$-linked scaffolds show a bimodal distribution with an increase of read mapping to female samples expected from the 2:1 ratio $\mathrm{X}$ chromosomes in females to males. This bimodality is not observed in autosomes. 
Supplementary Figure 5. The distribution of sex-based CpG fractional DNA methylation (5mC) differences across autosomes and $\mathrm{X}$ chromosomes.
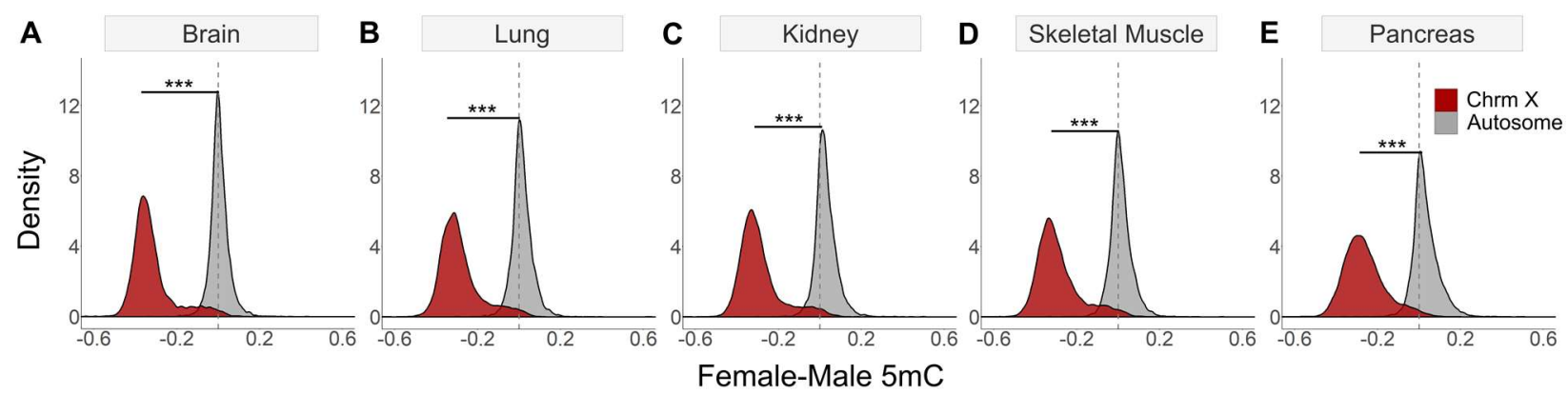

The distribution of female and male mean fractional methylation difference from (A) brain, (B) lung, (C) kidney, (D) skeletal muscle and (E) pancreas samples across autosomes and X chromosomes. For A-E, the female and male mean fractional methylation (methylated reads/total reads per $\mathrm{CpG}$ ) was calculated for all $\mathrm{CpGs}$ within $10 \mathrm{~kb}$ bins across each autosome- or X-linked scaffold. All tissues exhibited a significant shift towards female hypomethylation in the $\mathrm{X}$ chromosome compared to the autosome (*** indicates $\mathrm{p}<2.2 \times 10-16$, Welch's t-test). 
Supplementary Figure 6. Female and male gene expression across autosomes and the $\mathrm{X}$ chromosome using kidney RNA-seq data.

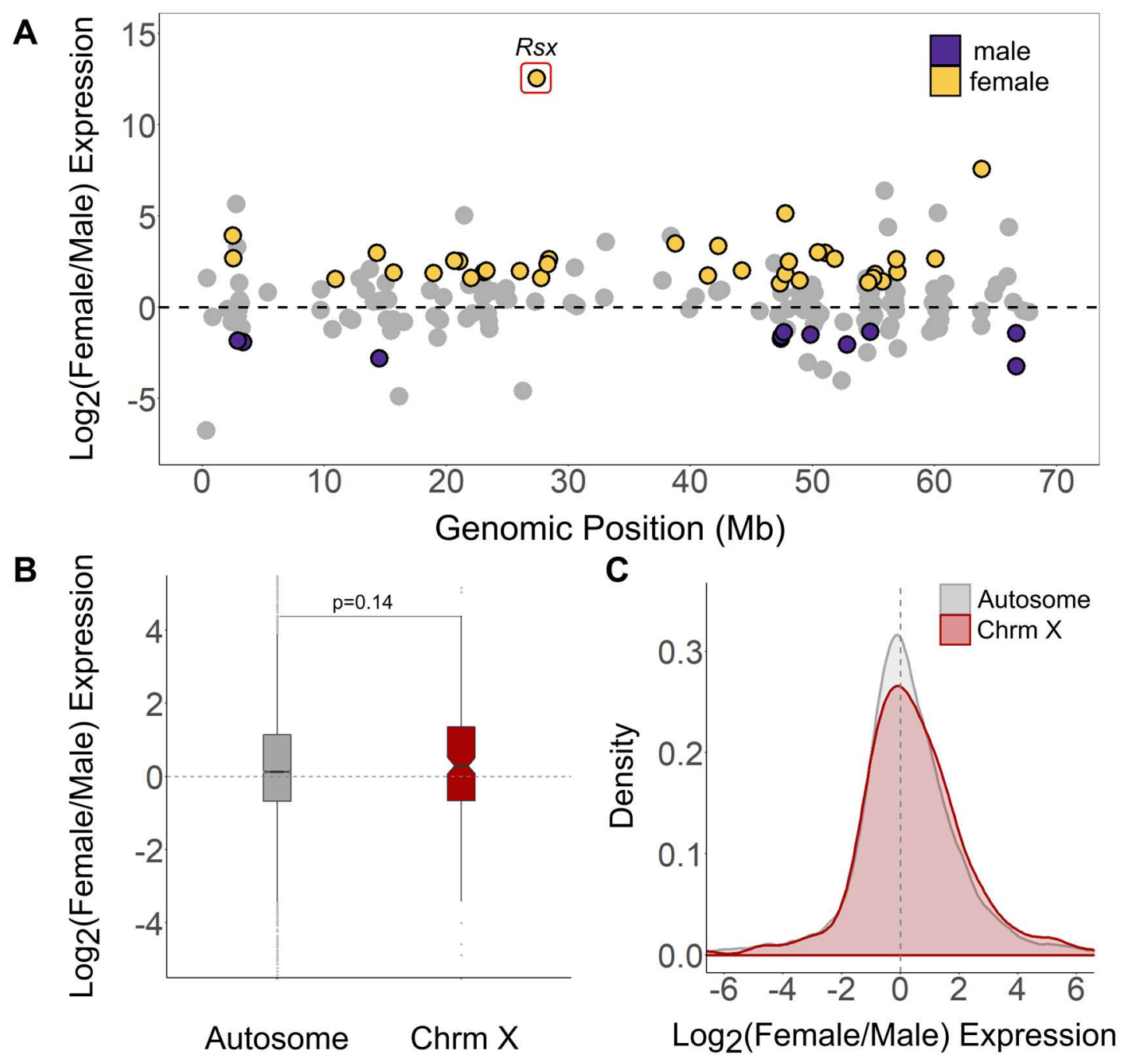

(A) The relative genomic location of all genes linked to $\mathrm{X}$ chromosome scaffolds aligned by scaffold length and the log-transformed female to male expression ratio generated by NOISeq. Orange dots indicate the 36 genes with significant female-biased expression and purple dots indicate the 11 genes with significant male-biased expression (probability of differential expression $>95 \%$ based on NOISeq). For all autosome-linked genes $(n=10,414)$ and chromosome X-linked genes ( $n=209)$, a box-and-whisker plot $(B)$ and density distribution (C) of the logtransformed female to male expression ratio $(\mathrm{p}=0.14$, Mann-Whitney $U$ test $)$. 
Supplementary Figure 7. Correlation analysis of sex-based DNA methylation $(5 \mathrm{mC})$ and gene expression across chromosome X using kidney WGBS and RNA-seq data.

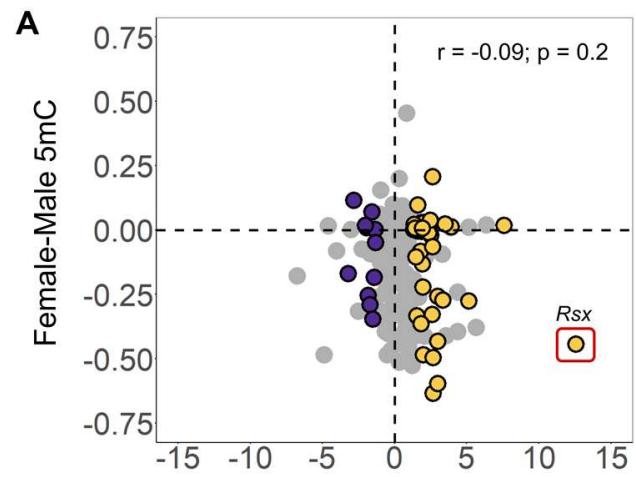

\begin{tabular}{|c|c|c|}
\cline { 2 - 3 } \multicolumn{1}{c|}{ B Promoter } & $\frac{\text { Male Over Expressed }}{\text { Total Genes }}$ & $\frac{\text { Female Over Expressed }}{\text { Total Genes }}$ \\
\hline $\begin{array}{c}\text { Female } \\
\text { Hypermethylated }\end{array}$ & $\frac{5}{32}=0.16$ & $\frac{14}{44}=0.32$ \\
\hline $\begin{array}{c}\text { Female } \\
\text { Hypomethylated }\end{array}$ & $\frac{6}{57}=0.11$ & $\frac{22}{73}=0.30$ \\
\hline
\end{tabular}

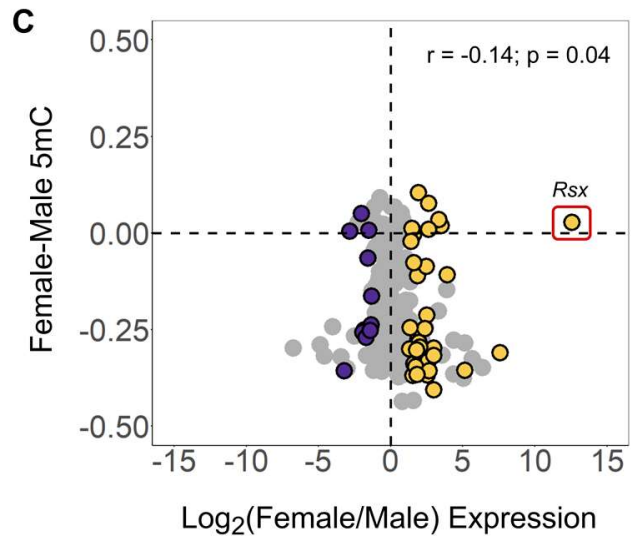

\begin{tabular}{|c|c|c|}
\cline { 2 - 3 } \multicolumn{1}{c|}{ Gene Body } & $\frac{\text { Male Over Expressed }}{\text { Total Genes }}$ & $\frac{\text { Female Over Expressed }}{\text { Total Genes }}$ \\
\hline $\begin{array}{c}\text { Female } \\
\text { Hypermethylated }\end{array}$ & $\frac{3}{11}=0.27$ & $\frac{7}{15}=0.47$ \\
\hline $\begin{array}{c}\text { Female } \\
\text { Hypomethylated }\end{array}$ & $\frac{8}{79}=0.10$ & $\frac{29}{103}=0.28$ \\
\hline
\end{tabular}

(A) The mean female and male fractional methylation difference calculated for all CpGs across each gene promoter matched with the corresponding log-transformed ratio of female to male expression. (B) The ratio of the number female hypermethylated and female hypomethylated gene promoters that show either significant male of female biased expression over the total number of genes in each category. (C) The mean female and male fractional methylation difference calculated for all $\mathrm{CpGs}$ across each gene body and matched with corresponding log-transformed ratio of female to male expression. (D) The ratio of the number female hypermethylated and female hypomethylated gene bodies that show either significant male of female biased expression over the total number of genes in each category. For A and C, Spearman's rank correlation coefficient and the associated p-value is reported. The Rsx gene was excluded from the correlation calculation. Orange dots indicate the 36 genes with significant female-biased expression and purple dots indicate the 11 genes with significant male-biased expression (probability of differential expression $>95 \%$ based on NOISeq). 
Supplementary Figure 8. Sex-based DNA methylation $(5 \mathrm{mC})$ by GC-content across autosomes and the $\mathrm{X}$ chromosome.
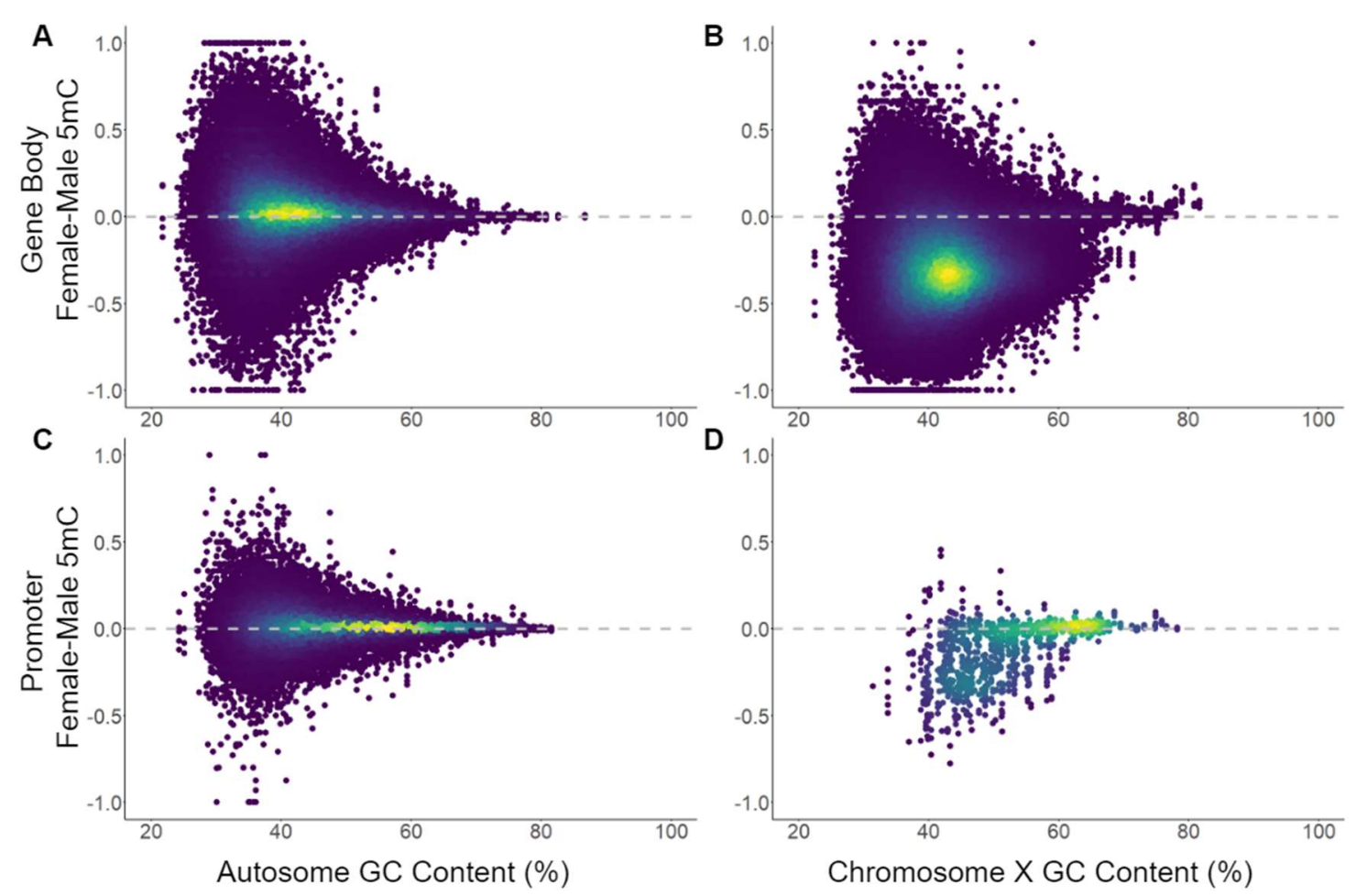

For $\mathrm{A}$ and $\mathrm{B}$, the mean female and male methylation difference calculated from CpGs in $1 \mathrm{~Kb}$ bins across (A) autosomes and (B) X chromosomes. For C and D, mean female and male methylation difference calculated from CpGs located in promoter regions (defined as regions $1 \mathrm{~kb}$ upstream of known gene TSSs) in (C) autosomes and (B) X chromosomes. For A-D, data from all five tissues (brain, kidney, lung, pancreas, and skeletal muscle) are reported. All plots are coloured by data density where blue represents low density regions and yellow represents high density regions. 